TRANSACTIONS OF THE

AMERICAN MATHEMATICAL SOCIETY

Volume 348, Number 12, December 1996, Pages 4939-4964

S 0002-9947(96)01605-4

\title{
A DEFORMATION OF FLAT CONFORMAL STRUCTURES
}

\author{
HIROYASU IZEKI
}

\begin{abstract}
We consider deformations of flat conformal structures from a viewpoint of connected sum decomposition of conformally flat manifolds.
\end{abstract}

\section{INTRODUCTION}

Let $M$ be a smooth $n$-manifold and $C$ a conformal class on $M$. If, for any point $p$ of $M$, there exists a metric $g$ in $C$ such that $g$ is flat on some neighborhood of $p$, then $(M, C)$ is called conformally flat. A conformal class on $M$ is called a flat conformal structure if $(M, C)$ is conformally flat. A Riemannian manifold $(M, g)$ is conformally flat if the conformal class containing $g$ is a flat conformal structure. And $M$ is said to be conformally flat if $M$ admits a flat conformal structure. If $n=2$ a flat conformal structure on an oriented surface $S$ is nothing but a conformal structure on $S$. A flat conformal structure on a conformally flat manifold is a natural generalization of a conformal structure on a surface. The space of conformal structures on an oriented surface is understood as, for instance, the Teichmüller space of Riemann surfaces. The Teichmüller space of a compact oriented surface $S$ with genus greater than one is identified with the quotient of the space of hyperbolic metrics on $S$ by the identity component of the diffeomorphism group of $S$. To understand this space, the following description is useful.

Let $(S, g)$ be a compact oriented surface of genus $h \geq 2$ with hyperbolic metric $g$. Then we can take $3 h-3$ disjoint simple closed geodesics with respect to $g$. Cutting $S$ along these geodesics, we obtain $2 h-2$ hyperbolic surfaces with geodesic boundaries, and these are homeomorphic to $S^{2}$ with three disks removed. These hyperbolic surfaces with geodesic boundaries are called pairs of (hyperbolic) pants. Hyperbolic structures on a pair of pants are determined by the lengths of the three boundaries. On the other hand, $S$ is obtained by gluing the boundaries of these pairs of pants by isometries. It is well-known that the lengths of the three boundaries and twisting parameters of identification of boundaries define coordinates on the Teichmüller space of $S$. In fact, it is easy to see that these coordinates consist of $6 h-6$ parameters (note that in order to identify boundaries they must have the same length), and it is well-known that the dimension of the Teichmüller space of a surface with genus $h$ is just $6 h-6$. The coordinates of Teichmüller space defined as above are called the Fenchel-Nielsen coordinates. We refer the reader to [2] for a detailed exposition.

The subject of this paper is an attempt to describe the space of flat conformal structures on a compact conformally flat $n$-manifold $(n \geq 3)$ in a way similar to the

Received by the editors December 16, 1994 and, in revised form, September 6, 1995.

1991 Mathematics Subject Classification. Primary 53A30.

(C)1996 American Mathematical Society 
description of the Teichmüller space in terms of the Fenchel-Nielsen coordinates. In order to realize such descriptions, we have to consider the following two problems:

1. Decompose a compact conformally flat manifold into small pieces with flat conformal structures.

2. Describe deformations of flat conformal structures by deformations of patching of these small pieces and deformations of flat conformal structures on these pieces.

In section 2, we consider Problem 1, using the $C$-prime decomposition (see section 2 or [8]) of compact conformally flat manifolds, which is a decomposition with respect to connected sums (recall that a connected sum of conformally flat manifolds is also conformally flat by a theorem of Kulkarni [12]). Then, in our case, a small piece corresponding to a pair of pants in Fenchel-Nielsen coordinates is a $C$ prime manifold (see Definition 2.1), which is a compact conformally flat manifold (without boundary). In order to obtain small pieces with flat conformal structures, since we decompose a manifold $M$ with respect to connected sums, such as $M=M_{1} \sharp M_{2}$, we have to extend a flat conformal structure on $M_{i} \backslash n$-disk, induced by restriction, to a flat conformal structure on $M_{i}(i=1,2)$. It is not known (to the author) whether this extension is possible or not in the general case. But for a compact manifold with Kleinian structure, this extention is possible and we obtain a reasonable decomposition with respect to connected sums.

Theorem 2.8. Let $M$ be a compact connected $n$-manifold with $n \geq 3$, and $C$ a Kleinian structure on $M$. Then there exist $C$-prime manifolds $P_{1}, \ldots, P_{k}$ and a Kleinian structure $C_{i}$ on $P_{i}(i=1, \ldots, k)$ such that $(M, C)$ is conformal to $\left(P_{1}, C_{1}\right) \sharp \ldots \sharp\left(P_{k}, C_{k}\right)$.

Here, $(M, C)$ is called Kleinian if $(M, C)$ is conformal to $\Omega / \Gamma$ for some open subset $\Omega$ of $S^{n}$ and some discrete subgroup $\Gamma$ of the conformal transformation group of standard $S^{n}$, which leaves $\Omega$ invariant and acts freely and properly discontinuously on $\Omega$. Note that $\Omega / \Gamma$ has a natural flat conformal structure. A conformal class $C$ on $M$ is called a Kleinian structure (or a uniformized conformal structure), if $(M, C)$ is Kleinian. See section 2 for the other notations. This theorem asserts that it is possible, in principle, to realize the description of the space of Kleinian structures in a way similar to the one using the Fenchel-Nielsen coordinates by means of the $C$-prime decomposition. In fact, we obtain a complete descriptiotion of the space of Kleinian structures on $\mathbf{R} P^{n} \sharp \mathbf{R} P^{n}$ in such a way (see Appendix). Since, in this particular case, any flat conformal structure is Kleinian, this gives us a complete description of the space of flat conformal structures on $\mathbf{R} P^{n} \sharp \mathbf{R} P^{n}$ following our program.

It should be mentioned that section 2 is based on a part of the author's previous paper [8], and can be considered as a supplement to [8].

In section 3, we construct a deformation of flat conformal structures on a connected sum of conformally flat manifolds from the viewpoint of Problem 2. Let $\left(M_{1}, g_{1}\right)$ and $\left(M_{2}, g_{2}\right)$ be compact conformally flat Riemannian manifolds and $p_{i}$ a point of $M_{i}(i=1,2)$. Then $\left(M_{i} \backslash\left\{p_{i}\right\},\left.g_{i}\right|_{M_{i} \backslash\left\{p_{i}\right\}}\right)$ is conformal to a complete Riemannian manifold whose end is isometric to a half-infinite cylinder $[0, \infty) \times S^{n-1}(1)$ with radius 1 . Cutting each end along the totally geodesic $(n-1)$-sphere of a halfinfinite cylinder and gluing the resulting manifolds along these $(n-1)$-spheres of boundaries by an isometry, we obtain $M_{1} \sharp M_{2}$ with a conformally flat metric. This Riemannian manifold contains a subset isometric to a finite cylinder $(0, l) \times S^{n-1}(1)$ 
for some positive real number $l$. Denote this Riemannian manifold by $\left(M_{1} \sharp M_{2}, \hat{g}_{l}\right)$. By cutting each end along another totally geodesic sphere in this process, we can construct a Riemannian manifold containing a finite cylinder of different length, say $l^{\prime}$. We denote this Riemannian manifold by $\left(M_{1} \sharp M_{2}, \hat{g}_{l^{\prime}}\right)$.

Theorem 3.1. If $l^{\prime}$ is greater than some constant depending only on $l, n, M_{1}$ and $M_{2}$, then $\left(M_{1} \sharp M_{2}, \hat{g}_{l}\right)$ and $\left(M_{1} \sharp M_{2}, \hat{g}_{l^{\prime}}\right)$ are not conformally equivalent, provided each $M_{i}$ is not diffeomorphic to $S^{n}$.

That is, by changing the length of a cylinder, we can deform a flat conformal structure on $M_{1} \sharp M_{2}$, if each $M_{i}$ is not diffeomorphic to $S^{n}$ (see section 3 for a more precise statement). On the other hand, if either $\left(M_{1}, g_{1}\right)$ or $\left(M_{2}, g_{2}\right)$ is conformal to the standard $S^{n}$, then two Riemannian manifolds constructed as above are always conformally equivalent (see Remark 2 following Theorem 3.1). As a consequence of Theorem 3.1, we see that the moduli space of flat conformal structures on a compact, non-trivial, conformally flat and non- $C$-prime manifold is noncompact (see Corollary 3.2). Here, the topology of the moduli space of $M$ is given by the quotient topology which comes from the compact $C^{\infty}$-topology of the space of all conformally flat Riemannian metrics on $M$.

The deformation above is related to the bending deformation of Thurston (see [20] and [5]). By Johnson and Millson, the bending deformations for compact manifolds are studied in terms of the cohomology of group representations in [9]. They proved a result corresponding to Theorem 3.1 for infinitesimal or small deformations, which says that the bending deformation gives rise to a non-trivial deformation of flat conformal structures in the Teichmüller space of $M_{1} \sharp M_{2}$ (section 5 of [9]). Here, the Teichmüller space of a manifold $M$ is the quotient of the space of all flat conformal structures on $M$ by the identity component of the group of diffeomorphisms of $M$. Theorem 3.1 tells us that the large bending deformation described above also gives us a non-trivial deformation in the moduli space, and we prove this by a purely geometric method. Here, we put special emphasis on the fact that our method also works for noncompact manifolds, if we consider the Teichmüller space instead of the moduli space, in contrast to the method of Johnson and Millson. See Theorem 3.4.

Though there are many other ways to deform connected sums defined in section 2, they do not always give us deformations of flat conformal structures (see Appendix). The result of Johnson and Millson mentioned above also suggests that, roughly speaking, a deformation of connected sums gives us a deformation of flat conformal structures on $M_{1} \sharp M_{2}$, if there is no conformal transformation on each $\left(M_{i}, C_{i}\right)$ which absorbs the deformation of connected sums. From this point of view, changing the length of a cylinder corresponds to essential conformal transformations, or more precisely, similarity transformations. And, as is expected from this, a theorem of Obata ([18]) and Lelong-Ferrand ([15], [16]) concerning the conformal transformation groups of compact Riemannian manifolds plays a key role in the proof of this theorem. Note that we do not restrict our attention to deformations of Kleinian structures here. See, for example, [1] for an exposition on deformations arising from those of discrete groups.

In the rest of this paper, we assume that a manifold $M$ is connected with $n=$ $\operatorname{dim} M \geq 3$. Moreover, if $M$ is orientable, we also assume that $M$ is oriented. 


\section{The $C$-PRIME DECOMPOSITION OF CONFORMALLY FLAT MANIFOLDS}

We start with a review of the $C$-prime decomposition of compact conformally flat manifolds defined in [8]. In this section, a manifold $M$ is always assumed to be compact.

Definition 2.1. An $n$-manifold $M$ is said to be non-trivial if $M$ is not diffeomorphic to $S^{n}$. And a non-trivial conformally flat manifold $M$ is called $C$-prime if $M$ is not diffeomorphic to a connected sum of non-trivial conformally flat manifolds.

Proposition 2.2 ([8, Proposition 2.1]). Every non-trivial conformally flat manifold is diffeomorphic to a connected sum $P_{1} \sharp \ldots \sharp P_{k}$ of a finite number of $C$-prime manifolds.

A decomposition $M=P_{1} \sharp \ldots \sharp P_{k}$, where each $P_{i}$ is $C$-prime, is called a $C$-prime decomposition of $M$. If $M$ is an oriented 3-manifold admitting a Kleinian structure, then this decomposition is unique (see [8, Corollary 3.7]). On the other hand, $N \sharp N$ is diffeomorphic to $N \sharp\left(S^{1} \times S^{2}\right)$, where $N$ is the non-orientable $S^{2}$-bundle over $S^{1}$ (see for example [7, Lemma 3.17]). Since the non-orientable $S^{2}$-bundle over $S^{1}$ and $S^{1} \times S^{2}$ are conformally flat and $C$-prime by Proposition 2.3 below, this is an example of a conformally flat manifold whose $C$-prime decomposition is not unique. It is not known whether the uniqueness holds for higher dimensional oriented manifolds.

Some sufficient conditions for a manifold to be $C$-prime are given as follows:

Proposition 2.3 ([8, Proposition 2.2]). If the fundamental group of a non-trivial conformally flat $n$-manifold $M$ is generated by one element, then $M$ is $C$-prime.

Proposition 2.4 ([8, Proposition 2.3]). If the universal covering space of a conformally flat manifold $M$ is diffeomorphic to $\mathbf{R}^{n}$, then $M$ is $C$-prime.

A manifold $M$ is said to be Kleinian if $M$ admits a Kleinian structure. For Kleinian manifolds, we have the following.

Proposition 2.5 ([8, Proposition 3.1]). Let $M$ be a non-trivial Kleinian manifold. If the homotopy group $\pi_{n-1}(M)$ is trivial, then $M$ is $C$-prime.

Take a subset $A$ of $S^{n}$ so that $A$ is diffeomorphic to $(0,1) \times S^{n-1}$ and satisfies the following conditions:

(1) Each connected component of $S^{n} \backslash A$ is diffeomorphic to a closed $n$-disk. Denote them by $D_{1}$ and $D_{2}$.

(2) $E_{i}=D_{i} \cup A(i=1,2)$ is diffeomorphic to the unit open $n$-disk and, under this identification, $D_{i}$ corresponds to the closed disk of radius $1 / 2$.

Fix an orientation of $S^{n}$. Let $C_{i}(i=1,2)$ be a flat conformal structure on $M_{i}$ and $\psi_{i}: E_{i} \longrightarrow\left(M_{i}, C_{i}\right)$ a conformal (orientation preserving) embedding. Note that $E_{i}$ has a natural flat conformal structure induced by restricting the flat conformal structure of $S^{n}$.

Definition 2.6. A connected sum $\left(M_{1}, C_{1}\right) \sharp\left(M_{2}, C_{2}\right)$ of $\left(M_{1}, C_{1}\right)$ and $\left(M_{2}, C_{2}\right)$ is defined by

$$
\left(M_{1} \backslash \psi_{1}\left(D_{1}\right),\left.C_{1}\right|_{M_{1} \backslash \psi_{1}\left(D_{1}\right)}\right) \cup_{\left.\psi_{2} \circ \psi_{1}^{-1}\right|_{\psi_{1}(A)}}\left(M_{2} \backslash \psi_{2}\left(D_{2}\right),\left.C_{2}\right|_{M_{2} \backslash \psi_{2}\left(D_{2}\right)}\right) .
$$

Remark. It is clear that this defines a flat conformal structure on $M_{1} \sharp M_{2}$ (moreover, if each $C_{i}$ is a Kleinian structure on $M_{i}$, then the flat conformal structure 
defined as above is also Kleinian by the proof of [13, Theorem 5.6], which states that a connected sum of Kleinian manifolds also admits a Kleinian structure). But this flat conformal structure depends on the choice of $A, \psi_{1}$ and $\psi_{2}$. Thus, more precisely speaking, we should denote the connected sum defined above by $\left(M_{1}, C_{1}\right) \sharp\left(A, \psi_{1}, \psi_{2}\right)\left(M_{2}, C_{2}\right)$ instead of $\left(M_{1}, C_{1}\right) \sharp\left(M_{2}, C_{2}\right)$. In fact, varying these choices, we can deform a flat conformal structure, as we will see in the next section. But, since it is not convenient to use $A, \psi_{1}$ and $\psi_{2}$ as parameters of flat conformal structures, we omit them in what follows.

Theorem 2.7. Let $(M, C)$ be Kleinian, and suppose $M$ is diffeomorphic to a connected sum $M_{1} \sharp M_{2}$, where $M_{1}$ and $M_{2}$ are not necessarily conformally flat. Then there exists a Kleinian manifold $M_{i}^{\prime}$, which is homeomorphic to $M_{i}$, and a Kleinian structure $C_{i}$ on $M_{i}^{\prime}(i=1,2)$ such that $(M, C)$ is conformal to some $\left(M_{1}^{\prime}, C_{1}\right) \sharp\left(M_{2}^{\prime}, C_{2}\right)$. If $n \leq 6, M_{i}^{\prime}$ is diffeomorphic to $M_{i}$.

Proof. The first assertion follows from the proof of [8, Theorem 3.2]. Let $S$ be a subset of $M$, which is an embedded $S^{n-1}$, such that $M \backslash S$ has two connected components, say $L_{i}$, where $L_{i}$ is diffeomorphic to $M_{i} \backslash n$-disk. Note that $L_{i}$ has a natural Kleinian structure induced by the restriction of a Kleinian structure $C$ of $M$. In the proof of [8, Theorem 3.2], this Kleinian structure is extended to a Kleinian structure on $M_{i}^{\prime}=L_{i} \cup n$-disk, and $M_{i}^{\prime}$ is clearly homeomorphic to $M_{i}$. Since $L_{i} \cup S$ is naturally embedded in $M_{i}$, if this embedding is extended to the $n$ disk, $M_{i}^{\prime}$ is diffeomorphic to $M_{i}$. If $n \leq 6$, it is possible to extend a diffeomorphism of the unit (n-1)-sphere of $\mathbf{R}^{n}$ to a diffeomorphism of the unit $n$-disk of $\mathbf{R}^{n}$ (see for example [3] and [17]). Applying this fact to the above embedding restricted to $S$, we see that the second assertion of the theorem follows.

Remark. We can not expect $M_{i}^{\prime}$ to be diffeomorphic to $M_{i}$ if $n \geq 7$. Let $M_{1}$ be an exotic $n$-sphere $\Sigma^{n}$ and $M_{2}$ be $\Sigma^{n}$ with the opposite orientation $(n \geq 7)$. Then $M=\Sigma^{n} \sharp\left(-\Sigma^{n}\right)$ is diffeomorphic to $S^{n}$ and clearly Kleinian, where $-\Sigma^{n}$ denotes $\Sigma^{n}$ with the opposite orientation. But $\Sigma^{n}$ does not admit a flat conformal structure. In this case, $M_{i}^{\prime}$ must be $S^{n}$.

Using Theorem 2.7, we can prove the following theorem mentioned in the introduction in the same manner as the proof of Proposition 2.2 (see [8, Proposition 2.1]).

Theorem 2.8. Let $(M, C)$ be Kleinain. Then there exist $C$-prime manifolds $P_{1}, \ldots$, $P_{k}$ and a Kleinian structure $C_{i}$ on $P_{i}(i=1, \ldots, k)$ such that $(M, C)$ is conformal to some $\left(P_{1}, C_{1}\right) \sharp \ldots \sharp\left(P_{k}, C_{k}\right)$.

Remark. Let $M$ be a Kleinian manifold, and fix a $C$-prime decomposition $M=$ $P_{1} \sharp \ldots \sharp P_{k}$ of $M$. If $n \leq 6$, we can decompose a Kleinian structure on $M$ with respect to this decomposition. That is, we can decompose $(M, C)$ as $\left(P_{1}, C_{1}\right) \sharp \ldots \sharp\left(P_{k}, C_{k}\right)$ for some Kleinian structure $C_{i}$ on $P_{i}$. We can not expect this for the case $n \geq 7$ at the present stage, since a differentiable structure of $P_{i}$ may change as we decompose a Kleinian structure $C$ on $M$.

In the rest of this section, we consider a certain sufficient condition for a manifold to be $C$-prime, and show that most of familiar examples of conformally flat manifolds are $C$-prime. Note that, for a Kleinian structure $C$ on $M$, there exists an open subset $\Omega$ of $S^{n}$ such that $(M, C)$ is conformal to a quotient of $\Omega$ by a discrete subgroup $\Gamma$ of the conformal transformation group of standard $S^{n}$ by definition. 
Proposition 2.9. Let $M$ be a Kleinian manifold and $C$ a Kleinian structure on $M$. If the number of connected components of $S^{n} \backslash \Omega$ is finite, then $M$ is either $C$-prime or diffeomorphic to $\mathbf{R} P^{n} \sharp \mathbf{R} P^{n}$.

Proof. Assume $M$ is not $C$-prime. Then, by Theorem 2.7, there exist non-trivial Kleinian manifolds $\left(M_{i}, C_{i}\right) \cong \Omega_{i} / \Gamma_{i}, i=1,2$, such that $(M, C)$ is conformal to a connected sum of $\left(M_{1}, C_{1}\right)$ and $\left(M_{2}, C_{2}\right)$. We show that unless the order of each $\Gamma_{i}$ is less than three, the number of connected components of $S^{n} \backslash \Omega$ is infinite. If the order of each $\Gamma_{i}$ is less than three, then each $\Omega_{i}$ must be compact and hence $\Omega_{i}=S^{n}$. Since $M_{i}$ is non-trivial, each $\Gamma_{i}$ is of order two. Thus each $M_{i}$ is diffeomorphic to the real projective space $\mathbf{R} P^{n}$.

Now we may assume that $\Gamma_{1}$ is of order at least three and $\Gamma_{2}$ is of order at least two. Thus $M_{1} \backslash n$-disk is covered by $\hat{\Omega}_{1}=\Omega_{1} \backslash$ (at least three disks) and $M_{2} \backslash n$-disk is covered by $\hat{\Omega}_{2}=\Omega_{2} \backslash$ (at least two disks). By the construction of $\Omega_{i}$ in the proof of [8, Theorem 3.2], we see that $\Omega$ is reconstructed by gluing copies of $\hat{\Omega}_{i}$ along the boundaries made by removing disks (this construction is the same as what Kulkarni and Pinkall used in the proof of [13, Theorem 5.6]). Denote these disks removed from $\Omega_{1}$ by $D_{1}, D_{2}, D_{3}, \ldots$, and disks removed from $\Omega_{2}$ by $E_{1}, E_{2}, \ldots$ (note that there are at least three $D_{i}$ 's and at least two $E_{i}$ 's). If $\Omega \cap D_{i}=D_{i}$, then either $M_{1}$ or $M_{2}$ must be diffeomorphic to $S^{n}$ as in the proof of Proposition 2.5 (see [8, Proposition 3.1]). Therefore $\Omega \cap D_{i} \neq D_{i}$ and hence $S^{n} \backslash \Omega$ has at least three connected components. Put $\hat{\Omega}_{2}$ into $D_{1}$ and glue their boundaries suitably. Then at least one removed disk $E_{i}$ remains, and in order to obtain $\Omega$, we have to put a copy of $\hat{\Omega}_{1}$ into it and glue them along their boundaries. Then we see that $\left(S^{n} \backslash \Omega\right) \cap D_{1}$ has at least two connected components. In order to construct $\Omega$, we have to repeat this process of putting copies of $\hat{\Omega}_{i}$ into $D_{1}$ infinitely. And when we put $k$ copies of $\hat{\Omega}_{1}$ into $D_{1}$, the number of the connected components of $\left(S^{n} \backslash \Omega\right) \cap D_{1}$ is at least $2^{k}$. Therefore $S^{n} \backslash \Omega$ has infinitely many connected components. This completes the proof.

Proposition 2.10. Let $M$ be a non-trivial manifold and not diffeomorphic to $\mathbf{R} P^{n} \sharp \mathbf{R} P^{n}$. If there exists a flat conformal structure $C$ such that $(M, C)$ is conformally covered by $S^{n}(1), \mathbf{R}^{n}, H^{n}(-1), \mathbf{R} \times S^{n-1}(1), \mathbf{R} \times H^{n-1}(-1)$, or $S^{p}(1) \times$ $H^{q}(-1)(p, q \geq 2)$, then $M$ is $C$-prime. Here $S^{m}(1)$ and $H^{m}(-1)$ denote the unit Euclidean $m$-sphere and hyperbolic $m$-space with constant curvature -1 respectively, and products are Riemannian products.

Proof. If $(M, C)$ is conformally covered by $\mathbf{R}^{n}, H^{n}(-1)$ or $\mathbf{R} \times H^{n-1}(-1)$ then the conclusion follows from Proposition 2.4. Since $\mathbf{R} \times S^{n-1}(1)$ and $S^{p}(1) \times H^{q}(-1)$ are conformally equivalent to $S^{n} \backslash$ (two points) and $S^{n} \backslash S^{q-1}$ respectively, we can apply Proposition 2.9 in this case.

Corollary 2.11. A non-trivial manifold admitting a locally homogeneous conformally flat metric is $C$-prime, except when it is $\mathbf{R} P^{n} \sharp \mathbf{R} P^{n}$.

Proof. If $(M, g)$ is locally homogeneous, then the Riemannian universal covering space $(\tilde{M}, \tilde{g})$ is homogeneous by a theorem of Singer $([19])$. Since the conformal transformation group $\operatorname{Conf}(\tilde{M}, \tilde{g})$ has positive dimension, either $(\tilde{M}, \tilde{g})$ is conformal to $S^{n}(1), \mathbf{R}^{n}, H^{n}(-1), \mathbf{R} \times S^{n-1}(1), \mathbf{R} \times H^{n-1}(-1)$, or $(\tilde{M}, \tilde{g})$ is conformally mapped onto $S^{n}(1) \backslash \Lambda$ by the developing map of $(\tilde{M}, \tilde{g})$, where $\Lambda$ is contained in a round $k$-sphere with $k \leq n-2$, by a theorem of Goldman and Kamishima ([6]). In 
the last case, $M$ is conformally equivalent to $\left(S^{n}(1) \backslash \Lambda\right) / \Gamma$ and $\Lambda$ is the limit set of a discrete subgroup $\Gamma$ of the conformal transformation group $\operatorname{Conf}\left(S^{n}\right)$ of the standard sphere. Let $S^{k}$ be a minimal round $k$-sphere containing $\Lambda$. Since if $k=0$, $S^{n}(1) \backslash \Lambda$ is conformal to $\mathbf{R} \times S^{n-1}(1)$ or $\mathbf{R}^{n}$, we may assume that $1 \leq k \leq n-2$. Let $G$ be the image of $\operatorname{Conf}(\tilde{M}, \tilde{g})$ by the map $\operatorname{Conf}(\tilde{M}, \tilde{g}) \longrightarrow \operatorname{Conf}\left(S^{n}\right)$ induced from the developing map of $(\tilde{M}, \tilde{g})$. Note that $G$ acts transitively on the developing image of $(\tilde{M}, \tilde{g})$ even if the developing map of $(\tilde{M}, \tilde{g})$ is not injective. It is clear that $G$ leaves the minimal round sphere $S^{k}$ invariant. Thus if $S^{k} \backslash \Lambda$ is not empty, $G$ can not act transitively on $S^{n}(1) \backslash \Lambda$, and hence the developing image of $\tilde{M}$ is $S^{n}(1) \backslash S^{k}$. Therefore $(M, g)$ is conformally covered by $S^{p}(1) \times H^{q}(-1)(p, q \geq 2)$ or $\mathbf{R} \times H^{n-1}(-1)$. This completes the proof.

\section{Constructing a Deformation}

In this section we prove Theorem 3.1 mentioned in the introduction. Before we prove Theorem 3.1, we give a more precise statement of the theorem.

Let $\left(M_{i}, g_{i}\right), i=1,2$, be a compact conformally flat Riemannian manifold, where each $M_{i}$ is not diffeomorphic to $S^{n}$. Let $d(M)$ be the smallest number of the generators of the fundamental group $\pi_{1}(M)$. We may assume $d\left(M_{1}\right) \geq d\left(M_{2}\right)$. Denote the integer part of $d\left(M_{1}\right) / d\left(M_{2}\right)$ by $k_{0}$. This number is well-defined since $d\left(M_{i}\right)$ is positive in our case by a theorem of Kuiper ([10]). Let $p_{i}$ be a point of $M_{i}$. Then $\left(M_{i} \backslash\left\{p_{i}\right\},\left.g_{i}\right|_{M_{i} \backslash\left\{p_{i}\right\}}\right)$ is conformal to a complete Riemannian manifold whose end is isometric to a half-infinite cylinder $[0, \infty) \times S^{n-1}(1)$. Denote them by $\left(\bar{M}_{i}, \bar{g}_{i}\right)$ and identify each end with $[0, \infty) \times S^{n-1}(1)$ by an isometry. Fix a positive real number $l$ and define $X=\left(\bar{M}_{1}, \bar{g}_{1}\right) \backslash[l, \infty) \times S^{n-1}(1)$ and $Y=\left(\bar{M}_{2}, \bar{g}_{2}\right) \backslash[l, \infty) \times$ $S^{n-1}(1)$ by using above identification of each end. Identifying $(0, l) \times S^{n-1} \subset X$ and $(0, l) \times S^{n-1} \subset Y$ by a suitable isometry, we obtain a compact conformally flat Riemannian manifold diffeomorphic to $M_{1} \sharp M_{2}$. Denote this by $\left(M, \hat{g}_{l}\right)$. Similarly, define

$$
\tilde{X}= \begin{cases}\left(\bar{M}_{1}, \bar{g}_{1}\right) \backslash\left[2 l+\left(k_{0}+1\right) l^{\prime}, \infty\right) \times S^{n-1}(1) & \text { if } d\left(M_{1}\right)>d\left(M_{2}\right), \\ \left(\bar{M}_{1}, \bar{g}_{1}\right) \backslash\left[2 l+3 l^{\prime}, \infty\right) \times S^{n-1}(1) & \text { if } d\left(M_{1}\right)=d\left(M_{2}\right),\end{cases}
$$

and

$$
\tilde{Y}= \begin{cases}\left(\bar{M}_{2}, \bar{g}_{2}\right) \backslash\left[2 l+\left(k_{0}+1\right) l^{\prime}, \infty\right) \times S^{n-1}(1) & \text { if } d\left(M_{1}\right)>d\left(M_{2}\right), \\ \left(\bar{M}_{2}, \bar{g}_{2}\right) \backslash\left[2 l+3 l^{\prime}, \infty\right) \times S^{n-1}(1) & \text { if } d\left(M_{1}\right)=d\left(M_{2}\right) .\end{cases}
$$

If $d\left(M_{1}\right)>d\left(M_{2}\right)$ (resp. $\left.d\left(M_{1}\right)=d\left(M_{2}\right)\right)$, by idendifying $\left(0,2 l+\left(k_{0}+1\right) l^{\prime}\right) \times$ $S^{n-1}(1) \subset \tilde{X}\left(\operatorname{resp} .\left(0,2 l+3 l^{\prime}\right) \times S^{n-1}(1) \subset \tilde{X}\right)$ with $\left(0,2 l+\left(k_{0}+1\right) l^{\prime}\right) \times S^{n-1}(1) \subset \tilde{Y}$ (resp. $\left.\left(0,2 l+3 l^{\prime}\right) \times S^{n-1}(1) \subset \tilde{Y}\right)$ suitably, we obtain a compact conformally flat Riemannian manifold diffeomorphic to $M$. Denote this by $\left(M, \hat{g}\left(l^{\prime}\right)\right)$ (see Figure 1). Now we can state Theorem 3.1.

Theorem 3.1. If $l^{\prime}$ is greater than a certain constant $C(n, l)$ depending only on $n$ and $l$, then $\left(M, \hat{g}_{l}\right)$ and $\left(M, \hat{g}\left(l^{\prime}\right)\right)$ are not conformally equivalent.

Remark 1. Let $C_{i}$ be a flat conformal structure on $M_{i}$ containing $g_{i}$, and $\hat{C}_{l}$ and $\hat{C}\left(l^{\prime}\right)$ flat conformal structures on $M$ containing $\hat{g}_{l}$ and $\hat{g}\left(l^{\prime}\right)$ respectively. Then it is clear that both $\left(M, \hat{C}_{l}\right)$ and $\left(M, \hat{C}\left(l^{\prime}\right)\right)$ are represented as $\left(M_{1}, C_{1}\right) \sharp\left(M_{2}, C_{2}\right)$, though these are not conformally equivalent. This is what we mentioned in the remark following Definition 2.6. 


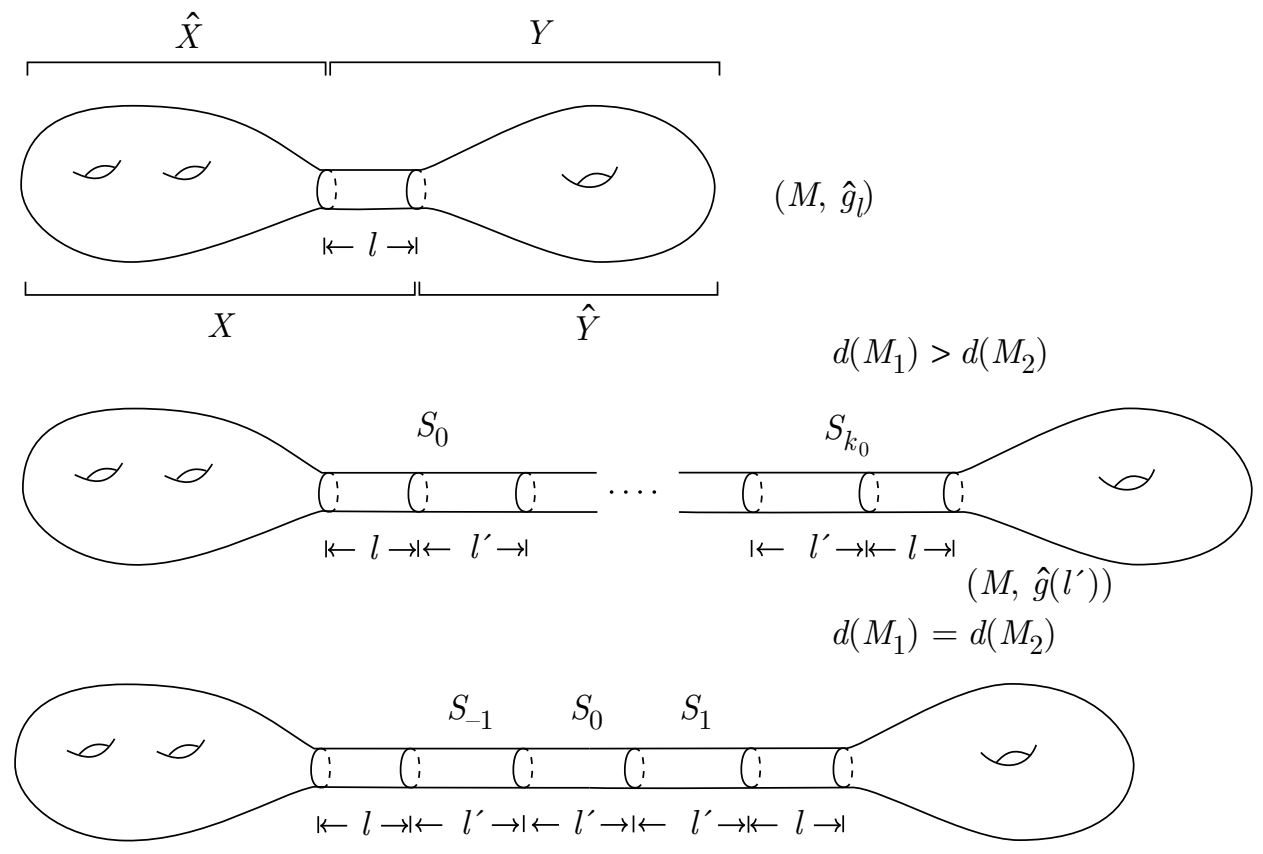

FiguRE 1

Remark 2. Assume $M_{2}$ is diffeomorphic to $S^{n}$. Then, by a theorem of Kuiper ([10]), $\left(M_{2}, g_{2}\right)$ is conformal to the standard $n$-sphere $S^{n}(1)$. In this case, $k_{0}$, and thus, $\left(M, \hat{g}\left(l^{\prime}\right)\right)$ can not be defined as above. But we can define $\left(M, \hat{g}_{l^{\prime \prime}}\right)$, which contains a subset isometric to $\left(0, l^{\prime \prime}\right) \times S^{n-1}(1)$ as follows: Choose $l^{\prime \prime}$ so that $l^{\prime \prime}>l$, and let $Y_{l^{\prime \prime}}=\left(\bar{M}_{2}, \bar{g}_{2}\right) \backslash\left[l^{\prime \prime}, \infty\right) \times S^{n-1}(1) \subset Y$. Identifying $\left(l^{\prime \prime}-l, l^{\prime \prime}\right) \times S^{n-1}(1) \subset Y_{l^{\prime \prime}}$ with $X \backslash \hat{X}$ by a suitable isometry, we obtain a conformally flat Riemannian manifold diffeomorphic to $M$. Denote this by $\left(M, g_{l^{\prime \prime}}\right)$. Note that there exists a conformal transformation $\psi: Y \longrightarrow Y_{l^{\prime \prime}}$ with $\psi\left((0, l) \times S^{n-1}(1)\right)=\left(l^{\prime \prime}-l, l^{\prime \prime}\right) \times S^{n-1}(1)$ by Liouville's theorem. Then it is easy to see that, by choosing such a conformal transformation suitably, we can construct a conformal diffeomorphism from $\left(M, \hat{g}_{l}\right)$ to $\left(M, \hat{g}_{l^{\prime \prime}}\right)$.

Assume that a compact conformally flat manifold $M$ is non-trivial and not $C$ prime. Then, by definition, there are conformally flat manifolds $M_{1}$ and $M_{2}$ such that $M$ is diffeomorphic to $M_{1} \sharp M_{2}$. Take a positive real number $l_{1}$. Define $l_{j}$, $j=1,2, \ldots$, inductively as follows. Choose $l_{j-1}^{\prime}$ to be greater than $C\left(n, l_{j-1}\right)$. Let $l_{j}=2 l_{j-1}+\left(k_{0}+1\right) l_{j-1}^{\prime}$ (resp. $\left.l_{j}=2 l_{j-1}+3 l_{j-1}^{\prime}\right)$, if $d\left(M_{1}\right)>d\left(M_{2}\right)$ (resp. $\left.d\left(M_{1}\right)=d\left(M_{2}\right)\right)$. Fix conformally flat Riemannian metrics $g_{1}$ and $g_{2}$ on $M_{1}$ and $M_{2}$ respectively, and a Riemannian metric $\hat{g}_{l_{j}}$ on $M$ as above. Then, by Theorem 3.1, $\left(M, \hat{g}_{l_{i}}\right)$ and $\left(M, \hat{g}_{l_{j}}\right)$ are not conformally equivalent, if $i \neq j$. Therefore, $\left\{\hat{g}_{l_{j}}\right\}_{j=1,2, \ldots}$ defines an infinite sequence in the moduli space of flat conformal structures on $M$. It is easy to see that this sequence in the moduli space is a divergent sequence. Thus we have the following corollary to Theorem 3.1. 
Corollary 3.2. If $M$ is compact, non-trival, conformally flat and not $C$-prime, then $M$ carries infinitely many different flat conformal structures. Moreover, the moduli space of flat conformal structures on $M$ is noncompact.

To prove Theorem 3.1, we need the following lemma.

Lemma 3.3. Suppose that there exists a conformal embedding $\psi$ of $\left(-\epsilon, l^{\prime}+\epsilon\right) \times$ $S^{n-1}(1)$ into $\left(M, \hat{g}_{l}\right), \epsilon>0$, and that $\left(M, \hat{g}_{l}\right) \backslash \psi\left(\left(0, l^{\prime}\right) \times S^{n-1}(1)\right)$ has two connected components. Then there exists a positive constant $C(n, l)$ with the following property: If $l^{\prime}>C(n, l)$, then there exists a closed interval $[\alpha, \beta] \subset\left(0, l^{\prime}\right)$ such that the closure of $\psi(A)$ is contained in either $X$ or $Y$, where $A=(\alpha, \beta) \times S^{n-1}(1)$.

The constant $C(n, l)$ in Lemma 3.3 is the same as $C(n, l)$ in Theorem 3.1. The proof of Lemma 3.3 is given in the next section. We note here that we do not have to assume $M$ to be compact in Lemma 3.3.

Proof of Theorem 3.1. Take $l^{\prime}$ so that $l^{\prime}>C(n, l)$. Assume that there exists a conformal diffeomorphism $\varphi:\left(M, \hat{g}\left(l^{\prime}\right)\right) \longrightarrow\left(M, \hat{g}_{l}\right)$, that is, $\left(M, \hat{g}_{l}\right)$ and $\left(M, \hat{g}\left(l^{\prime}\right)\right)$ are conformally equivalent. We prove the theorem by showing that this assumption leads to a contradiction.

Define subsets $S_{k}$ of $\left(M, \hat{g}\left(l^{\prime}\right)\right)$ by

$$
S_{k}=\left\{\begin{array}{lc}
{\left[l+k l^{\prime}, l+(k+1) l^{\prime}\right] \times S^{n-1}(1) \subset \tilde{X},} & 0 \leq k \leq k_{0} \\
{\left[l+(k+1) l^{\prime}, l+(k+2) l^{\prime}\right] \times S^{n-1}(1) \subset \tilde{X},} & \text { if } d\left(M_{1}\right)>d\left(M_{2}\right), \\
& \text { if } d\left(M_{1}\right)=d\left(M_{2}\right) .
\end{array}\right.
$$

The natural inclusion $X \longrightarrow \tilde{X}$ (resp. $Y \longrightarrow \tilde{Y}$ ) defines an isometric embedding $i_{X}: X \longrightarrow\left(M, \hat{g}\left(l^{\prime}\right)\right)$ (resp. $\left.i_{Y}: Y \longrightarrow\left(M, \hat{g}\left(l^{\prime}\right)\right)\right)$. Define $\hat{X}$ and $\hat{Y}$ by $\hat{X}=$ $\left(M, \hat{g}_{l}\right) \backslash Y \subset X$ and $\hat{Y}=\left(M, \hat{g}_{l}\right) \backslash X \subset Y$.

Clearly, a restriction of $\varphi$ on some neighborhood of $S_{0}$ satisfies the assumption of Lemma 3.3. Thus we can take a subset $A_{0}$ of $S_{0}$ as $A$ in the lemma. Then $\left(M, \hat{g}\left(l^{\prime}\right)\right) \backslash A_{0}$ has two connected components, and we denote them by $\tilde{X}_{0}$ and $\tilde{Y}_{0}$ so that $\tilde{X}_{0} \supset i_{X}(X)$ and $\tilde{Y}_{0} \supset i_{Y}(Y)$. By our choice of $A_{0}, \varphi\left(A_{0}\right)$ is contained in either $X$ or $Y$. If $\varphi\left(A_{0}\right) \subset X$, then one out of two connected components of $\left(M, \hat{g}_{l}\right) \backslash \varphi\left(A_{0}\right)$ is contained in $X$, i.e., either $\varphi\left(\tilde{X}_{0}\right) \subset X$ or $\varphi\left(\tilde{Y}_{0}\right) \subset X$ holds. If $\varphi\left(A_{0}\right) \subset Y$, then either $\varphi\left(\tilde{X}_{0}\right) \subset Y$ or $\varphi\left(\tilde{Y}_{0}\right) \subset Y$ holds.

We divide the rest of the proof into several steps and label them as (3.k.m). The first " 3 " stands for the number of this section, the second " $k$ " stands for $k$ of $S_{k}$ and the last " $m$ " denotes a number of possible cases which occur as we consider the embedding of $S_{k}$ into $\left(M, \hat{g}_{l}\right)$ (these cases are listed in (3.1.3)).

(3.0.1): $\varphi\left(A_{0}\right) \subset X$ and $\varphi\left(\tilde{X}_{0}\right) \subset X$. In this case, there is a conformal embedding

$$
i_{X} \circ \varphi: \tilde{X}_{0} \cup A_{0} \longrightarrow \tilde{X}_{0} \cup A_{0}
$$


and $i_{X} \circ \varphi\left(\tilde{X}_{0} \cup A_{0}\right) \subset i_{X}(X)$. Since $\tilde{X}_{0} \cup A_{0}$ is conformal to $\left(M_{1}, g_{1}\right)$ with a disk removed, we regard $\tilde{X}_{0} \cup A_{0}$ as a subset of $\left(M_{1}, g_{1}\right)$. Define $D_{0}, D_{1}, D_{2}$ and $D_{3}$ by

$$
\begin{aligned}
& D_{0}=\left(M_{1}, g_{1}\right) \backslash\left(\tilde{X}_{0} \cup A_{0}\right), \\
& D_{1}=\left(M_{1}, g_{1}\right) \backslash \tilde{X}_{0}, \\
& D_{2}=\left(M_{1}, g_{1}\right) \backslash i_{X} \circ \varphi\left(\tilde{X}_{0} \cup A_{0}\right), \\
& D_{3}=\left(M_{1}, g_{1}\right) \backslash i_{X} \circ \varphi\left(\tilde{X}_{0}\right) .
\end{aligned}
$$

Then we have $D_{0} \subset D_{1} \subset D_{2} \subset D_{3}$. Since we take $A_{0}$ as in Lemma 3.3, $A_{0}$ is conformal to $(\alpha, \beta) \times S^{n-1}(1)$. Therefore $i_{X} \circ \varphi\left(A_{0}\right)$ is identified conformally with an open subset $S\left(\alpha_{0}\right)$ of $S^{n}(1)$ for some $\alpha_{0}>0$, where

$$
S\left(\alpha_{0}\right)=\left\{x=\left(x_{0}, \ldots, x_{n}\right) \in S^{n}(1) \subset \mathbf{R}^{n+1} ;\left|x_{0}\right|<\alpha_{0}\right\} .
$$

We may assume that $\partial\left(i_{X} \circ \varphi\left(\tilde{X}_{0} \cup A_{0}\right)\right)$ is identified with $\left\{x \in S^{n} ; x_{0}=\alpha_{0}\right\}$, where $\partial\left(i_{X} \circ \varphi\left(\tilde{X}_{0} \cup A_{0}\right)\right)$ denotes the boundary of $i_{X} \circ \varphi\left(\tilde{X}_{0} \cup A_{0}\right)$. Denote two connected components of $S^{n} \backslash S\left(\alpha_{0}\right)$ by $E\left(-\alpha_{0}\right)$ and $E\left(\alpha_{0}\right)$ so that $\partial E\left(\alpha_{0}\right)=\{x \in$ $\left.S^{n} ; x_{0}=\alpha_{0}\right\}$. By the above conformal identification of $i_{X} \circ \varphi\left(A_{0}\right)$ and $S\left(\alpha_{0}\right)$, we can glue $S\left(\alpha_{0}\right) \cup E\left(\alpha_{0}\right)$ to $\left(M_{1}, g_{1}\right) \backslash D_{2}=i_{X} \circ \varphi\left(\tilde{X}_{0} \cup A_{0}\right)$ and obtain a compact conformally flat manifold $M_{1}^{\prime}$. Similarly, we can glue $S\left(\alpha_{0}\right) \cup E\left(-\alpha_{0}\right)$ to $D_{3}$ and obtain a compact conformally flat manifold $M_{2}^{\prime}$. Clearly $M_{1}$ is diffeomorphic to $M_{1}^{\prime} \sharp M_{2}^{\prime}$ and $\pi_{1}\left(M_{1}\right) \cong \pi\left(M_{1}^{\prime}\right)$. Thus $\pi_{1}\left(M_{2}^{\prime}\right)=1$ by van Kampen's theorem, and hence $M_{2}^{\prime}$ is conformal to $S^{n}$ by a theorem of Kuiper ([10]). Therefore we have a conformal embedding $\tau: D_{3} \longrightarrow S^{n}$, and $\left.i_{X} \circ \varphi\right|_{A_{0}}$ defines a locally defined conformal diffeomorphism $\sigma: \tau\left(A_{0}\right) \longrightarrow \tau\left(i_{X} \circ \varphi\left(A_{0}\right)\right)$. By Liouville's theorem $\sigma$ is extended to a globally defined conformal transformation $\tilde{\sigma}$ on $S^{n}$. Note that $\tilde{\sigma}$ satisfies $\tilde{\sigma} \circ \tau\left(A_{0}\right)=\tilde{\sigma} \circ \tau\left(D_{1} \backslash D_{0}\right)=\tau\left(D_{3} \backslash D_{2}\right)$ and, in particular, $\tilde{\sigma} \circ \tau\left(D_{0}\right)=\tau\left(D_{2}\right)$ and $\tilde{\sigma} \circ \tau\left(D_{1}\right)=\tau\left(D_{3}\right)$. Define

$$
\eta= \begin{cases}i_{X} \circ \varphi & \text { on } \tilde{X}_{0} \cup A_{0}, \\ \tau^{-1} \circ \tilde{\sigma} \circ \tau & \text { on } D_{1} .\end{cases}
$$

Then $\eta$ is a well-defined conformal transformation on $\left(M_{1}, g_{1}\right)$. In particular, $\eta\left(D_{1}\right)=D_{3}$. But since $D_{3} \backslash i_{X} \circ \varphi\left(A_{0}\right) \supset D_{1}, D_{1}$ and $D_{3}$ never have the same volume with respect to any Riemannian metric on $M_{1}$. That is, $\eta$ never be an isometry with respect to any Riemannian metric on $M_{1}$. Therefore $\eta$ is an essential conformal transformation of $\left(M_{1}, g_{1}\right)$. Thus, by a theorem of Obata $([18])$ and Lelong-Ferrand ([15], [16]), $\left(M_{1}, g_{1}\right)$ is conformal to $S^{n}(1)$. A contradiction.

(3.0.2): $\varphi\left(A_{0}\right) \subset Y$. In this case, either $\varphi\left(\tilde{X}_{0}\right) \subset Y$ or $\varphi\left(\tilde{Y}_{0}\right) \subset Y$ holds. If $\varphi\left(\tilde{Y}_{0}\right) \subset$ $Y$, then this contradicts our assumption for the same reason as in (3.0.1). Assume $\varphi\left(\tilde{X}_{0}\right) \subset Y$. By regarding $Y$ as a subset of $\left(M_{2}, g_{2}\right)$, we see that $\varphi\left(\tilde{X}_{0} \cup A_{0}\right)$ $\subset\left(M_{2}, g_{2}\right)$. Then as we have seen in (3.0.1), we obtain compact conformally flat manifolds $M^{\prime}$ and $M_{1}^{\prime}$ such that $M_{2}=M_{1}^{\prime} \sharp M^{\prime}$ and $\pi_{1}\left(M_{1}^{\prime}\right) \cong \pi_{1}\left(M_{1}\right)$. By van Kampen's theorem and the Gruško-Neumann theorem (see for example [14, p. 58]), we see that $d\left(M_{2}\right)=d\left(M_{1}\right)+d\left(M^{\prime}\right)$. If $d\left(M_{1}\right)>d\left(M_{2}\right)$, we have a contradiction. If $d\left(M_{1}\right)=d\left(M_{2}\right)$, replace a number " $i$ " of $M_{i}$ and rename suitably $X, Y, \tilde{X}, \tilde{Y}$, and so on. Then, $\varphi\left(A_{0}\right) \subset X$ and $\varphi\left(\tilde{Y}_{0}\right) \subset X$ hold. This is the case that we consider in the next step. 

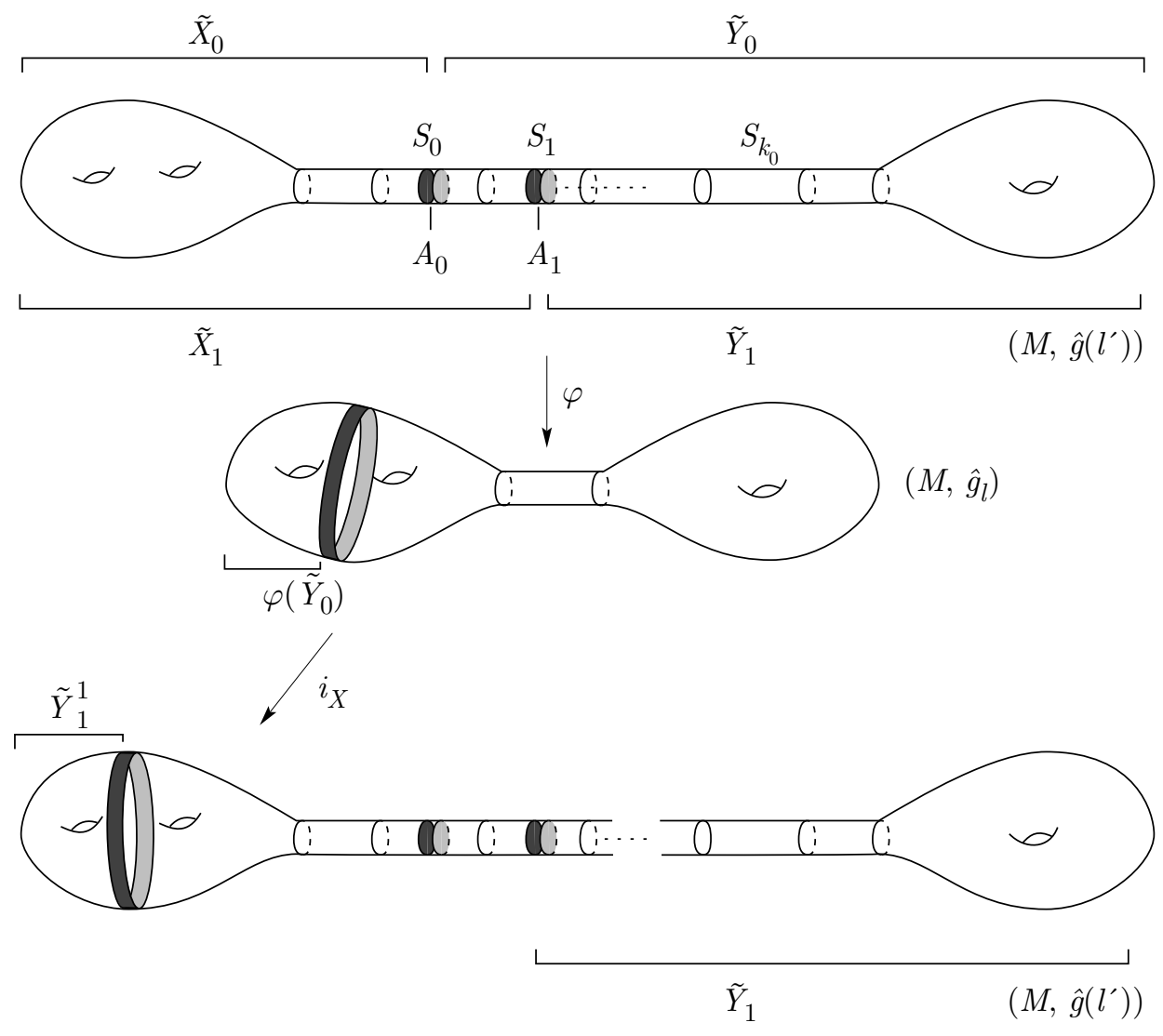

FiguRE 2

(3.0.3): $\varphi\left(A_{0}\right) \subset X$ and $\varphi\left(\tilde{Y}_{0}\right) \subset X$. Since $\tilde{Y}_{0} \supset S_{1}$ and $\varphi\left(\tilde{Y}_{0}\right) \subset X$, we can define $i_{X} \circ \varphi$ on a neighborhood of $S_{1}$. Then it is easy to see that the conformal embedding $\varphi \circ i_{X} \circ \varphi$ : (a neighborhood of $\left.S_{1}\right) \longrightarrow\left(M, \hat{g}_{l}\right)$ satisfies the assumption of Lemma 3.3. Take $A_{1} \subset S_{1}$ as in Lemma 3.3. Denote two connected components of $\left(M, \hat{g}\left(l^{\prime}\right)\right) \backslash A_{1}$ by $\tilde{X}_{1}$ and $\tilde{Y}_{1}$ so that $\tilde{X}_{1} \supset i_{X}(X)$ and $\tilde{Y}_{1} \supset i_{Y}(Y)$. Note that $\tilde{X}_{0} \subset \tilde{X}_{1}, \tilde{Y}_{0} \supset \tilde{Y}_{1}$. Since $\varphi\left(\tilde{Y}_{1} \cup A_{1}\right) \subset \varphi\left(\tilde{Y}_{0}\right) \subset X$, we can define $i_{X} \circ \varphi\left(A_{1}\right)$ and $i_{X} \circ \varphi\left(\tilde{Y}_{1}\right)$. Denote them by $A_{1}^{1}$ and $\tilde{Y}_{1}^{1}$, respectively. Note that $\left(M, \hat{g}\left(l^{\prime}\right)\right) \backslash A_{1}^{1}$ consists of two connected components $\tilde{Y}_{1}^{1}$ and $\left(M, \hat{g}\left(l^{\prime}\right)\right) \backslash\left(\tilde{Y}_{1}^{1} \cup A_{1}^{1}\right)$. See Figure 2 .

(3.1.1): $\varphi\left(A_{1}^{1}\right) \subset X$ and $\varphi\left(\left(M, \hat{g}\left(l^{\prime}\right)\right) \backslash\left(\tilde{Y}_{1}^{1} \cup A_{1}^{1}\right)\right) \subset X$. In this case, $\varphi\left(\tilde{Y}_{1}^{1}\right) \supset$ $\left(\left(M, \hat{g}_{l}\right) \backslash X\right)=\hat{Y}$. By the argument in (3.k.1) below, we see that this case does not occur.

(3.1.2): $\varphi\left(A_{1}^{1}\right) \subset Y$. By the argument in (3.k.2) below, we see that this contradicts our assumption.

(3.1.3): $\varphi\left(A_{1}^{1}\right) \subset X$ and $\varphi\left(\tilde{Y}_{1}^{1}\right)=\varphi \circ i_{X} \circ \varphi\left(\tilde{Y}_{1}\right) \subset X$. Since $S_{2} \subset \tilde{Y}_{1}$, we can define $\left(i_{X} \circ \varphi\right)^{2}$ on a neighborhood of $S_{2}$. As in (3.0.3), the conformal embedding $\varphi \circ$ $\left(i_{X} \circ \varphi\right)^{2}:\left(\right.$ a neighborhood of $\left.S_{2}\right) \longrightarrow\left(M, \hat{g}_{l}\right)$ satisfies the assumption of Lemma 
3.3, and hence we can take $A_{2} \subset S_{2}$ as in the lemma. Denote $\left(i_{X} \circ \varphi\right)^{2}\left(A_{2}\right)$ by $A_{2}^{2}$, and two connected components of $\left(M, \hat{g}\left(l^{\prime}\right)\right) \backslash A_{2}$ by $\tilde{X}_{2}$ and $\tilde{Y}_{2}$ so that $\tilde{X}_{2} \supset i_{X}(X)$ and $\tilde{Y}_{2} \supset i_{Y}(Y)$. Since $\varphi \circ i_{X} \circ \varphi\left(\tilde{Y}_{2}\right) \subset \varphi \circ i_{X} \circ \varphi\left(\tilde{Y}_{1}\right) \subset X,\left(i_{X} \circ \varphi\right)^{2}$ is defined on $\tilde{Y}_{2}$. Let $\tilde{Y}_{2}^{2}=\left(i_{X} \circ \varphi\right)^{2}\left(\tilde{Y}_{2}\right)$. Then $\left(M, \hat{g}\left(l^{\prime}\right)\right) \backslash A_{2}^{2}$ has two connected components $\tilde{Y}_{2}^{2}$ and $\left(M, \hat{g}\left(l^{\prime}\right)\right) \backslash\left(\tilde{Y}_{2}^{2} \cup A_{2}^{2}\right)$. In general, if $\varphi \circ\left(i_{X} \circ \varphi\right)^{j}\left(\right.$ a neighborhood of $\left.S_{k}\right) \subset X$ holds for $j$ with $0 \leq j \leq k-1$, then $\left(i_{X} \circ \varphi\right)^{k}$ is defined on a neighborhood of $S_{k}$ and the conformal embedding

$$
\left.\varphi \circ\left(i_{X} \circ \varphi\right)^{k}: \text { (a neighborhood of } S_{k}\right) \longrightarrow\left(M, \hat{g}_{l}\right)
$$

satisfies the assumption of Lemma 3.3. Thus we can take $A_{k} \subset S_{k}$ so that $\varphi \circ$ $\left(i_{X} \circ \varphi\right)^{k}\left(A_{k}\right)$ is contained in either $X$ or $Y$. Denote two connected components of $\left(M, \hat{g}\left(l^{\prime}\right)\right) \backslash A_{k}$ by $\tilde{X}_{k}$ and $\tilde{Y}_{k}$ so that $\tilde{X}_{k} \supset i_{X}(X)$ and $\tilde{Y}_{k} \supset i_{Y}(Y)$. Define $A_{k}^{j}$ by

$$
A_{k}^{j}=\left(i_{X} \circ \varphi\right)^{j}\left(A_{k}\right)
$$

and let $A_{k}^{0}$ be $A_{k}$. If we can take $A_{j}$ and $A_{k}$ with $j \leq k$, then $\tilde{Y}_{j} \supset \tilde{Y}_{k}$. Define $\tilde{Y}_{k}^{j}$ by

$$
\tilde{Y}_{k}^{j}=\left(i_{X} \circ \varphi\right)^{j}\left(\tilde{Y}_{k}\right)
$$

and let $\tilde{Y}_{k}^{0}$ be $\tilde{Y}_{k}$ when $\left(i_{X} \circ \varphi\right)^{j}$ is defined on $\tilde{Y}_{k}$, i.e., $\varphi \circ\left(i_{X} \circ \varphi\right)^{i}\left(\tilde{Y}_{k}\right) \subset X$ for $i$ with $0 \leq i \leq j-1$. If $A_{k}^{j}$ and $\tilde{Y}_{k}^{j}$ are defined, then $\left(M, \hat{g}\left(l^{\prime}\right)\right) \backslash A_{k}^{j}$ has two connected components and they are $\tilde{Y}_{k}^{j}$ and $\left(M, \hat{g}\left(l^{\prime}\right)\right) \backslash\left(\tilde{Y}_{k}^{j} \cup A_{k}^{j}\right)$. Note that $\left(\tilde{Y}_{k}^{j} \cup A_{k}^{j}\right) \subset \tilde{X}_{k}$ in this case, since $\varphi\left(A_{k}^{j-1} \cup \tilde{Y}_{k}^{j-1}\right) \subset X$. In the case that $A_{k}^{k}$ and $\tilde{Y}_{k}^{k}$ are defined, $\varphi\left(A_{k}^{k}\right)$ is contained in either $X$ or $Y$ by the definition of $A_{k}$, and there are three possibilities:

(1) $\varphi\left(A_{k}^{k}\right) \subset X$ and $\varphi\left(\tilde{Y}_{k}^{k}\right) \supset \hat{Y}$.

(2) $\varphi\left(A_{k}^{k}\right) \subset Y$.

(3) $\varphi\left(A_{k}^{k}\right) \subset X$ and $\varphi\left(\tilde{Y}_{k}^{k}\right) \subset X$.

In the case $k=0$ and either (1) or (2) holds, we have seen, in (3.0.1) and (3.0.2), that this contradicts our assumption. If $k=0$ and (3) holds, $A_{1}^{1}$ and $\tilde{Y}_{1}^{1}$ are defined and they satisfy (1), (2) or (3) with $k=1$ as we have seen in (3.0.3). If either (1) or (2) holds, as we have mentioned in (3.1.1) and (3.1.2), then we can derive a contradiction by (3.k.1) or (3.k.2) below with $k=1$. If (3) holds, $A_{2}^{2}$ and $\tilde{Y}_{2}^{2}$ are defined. In general, if (3) holds for $k$ with $0 \leq k \leq j_{0}-1$, then, since $\tilde{Y}_{k} \supset \tilde{Y}_{j_{0}} \cup S_{j_{0}}$ and $\varphi\left(\tilde{Y}_{k}^{k}\right) \subset X, \tilde{Y}_{j_{0}}^{j}$ and $A_{j_{0}}^{j}$ are defined for $0 \leq j \leq j_{0}$ and one of the above three possibilities holds for $k=j_{0}$. Let $k^{\prime}$ be the smallest number for which (3) does not hold. Then (1) or (2) holds for $k=k^{\prime}$. We have already seen the case $k^{\prime}=1$. In the case when (1) holds for $k^{\prime} \geq 2$, since $\varphi\left(A_{k^{\prime}}^{j} \cup \tilde{Y}_{k^{\prime}}^{j}\right) \subset \varphi\left(\tilde{Y}_{j}^{j}\right) \subset X$, we can derive a contradiction by (3.k.1) below. In the case when (2) holds for $k^{\prime} \geq 2$, we can derive a contradiction by $(3 . k .2)$ below.

Now assume that (3) holds for any $k\left(0 \leq k \leq k_{0}\right)$. Since $\tilde{Y}_{k} \supset \tilde{Y}_{k_{0}} \cup A_{k_{0}}$ for $k$, $0 \leq k \leq k_{0}-1$, we have $\varphi \circ\left(i_{X} \circ \varphi\right)^{k}\left(\tilde{Y}_{k_{0}} \cup A_{k_{0}}\right) \subset X$ for any $k$. Thus

$$
\tilde{X}_{k_{0}} \supset \bigcup_{k=0}^{k_{0}}\left(i_{X} \circ \varphi\right)^{k+1}\left(\tilde{Y}_{k_{0}} \cup A_{k_{0}}\right)=\bigcup_{k=1}^{k_{0}+1}\left(\tilde{Y}_{k_{0}}^{k} \cup A_{k_{0}}^{k}\right) \text {. }
$$

Since $\varphi\left(\tilde{Y}_{k_{0}} \cup A_{k_{0}}\right) \subset X, i_{X} \circ \varphi\left(\tilde{Y}_{k_{0}} \cup A_{k_{0}}\right)=\left(\tilde{Y}_{k_{0}}^{1} \cup A_{k_{0}}^{1}\right)$ is contained in $\tilde{X}_{k_{0}}$. Thus $\left(\tilde{Y}_{k_{0}}^{1} \cup A_{k_{0}}^{1}\right) \cap\left(\tilde{Y}_{k_{0}} \cup A_{k_{0}}\right)=\emptyset$ and hence $\varphi\left(\tilde{Y}_{k_{0}}^{1} \cup A_{k_{0}}^{1}\right) \cap \varphi\left(\tilde{Y}_{k_{0}} \cup A_{k_{0}}\right)=\emptyset$. Since $\varphi\left(\tilde{Y}_{k_{0}}^{1} \cup A_{k_{0}}^{1}\right) \subset X,\left(\tilde{Y}_{k_{0}}^{2} \cup A_{k_{0}}^{2}\right)=i_{X} \circ \varphi\left(\tilde{Y}_{k_{0}}^{1} \cup A_{k_{0}}^{1}\right)$ is contained in $\tilde{X}_{k_{0}}$, and hence 

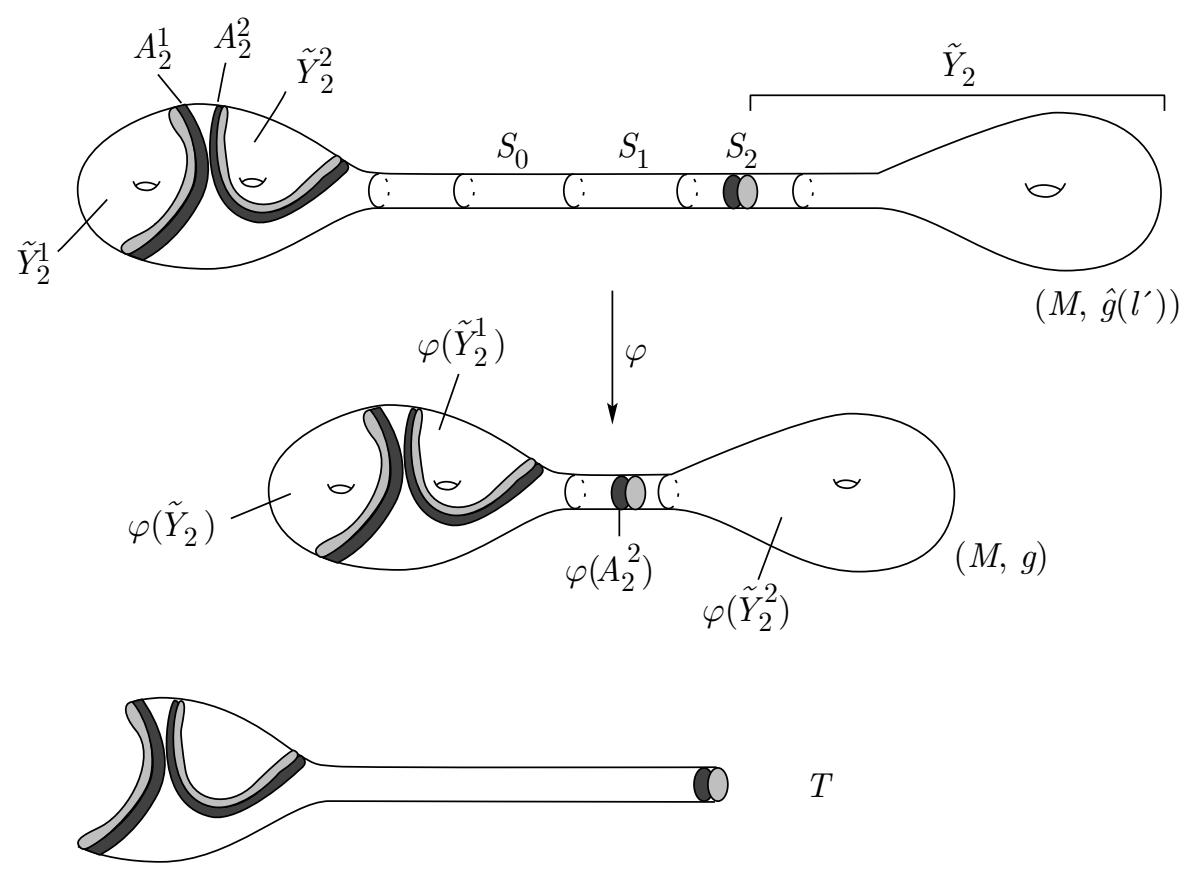

Figure 3

$\tilde{Y}_{k_{0}}^{2} \cup A_{k_{0}}^{2}, \tilde{Y}_{k_{0}}^{1} \cup A_{k_{0}}^{1}$ and $\tilde{Y}_{k_{0}} \cup A_{k_{0}}$ are disjoint. Proceeding this way, we see that $\tilde{Y}_{k_{0}}^{k} \cup A_{k_{0}}^{k}\left(k=0, \ldots, k_{0}+1\right)$ are mutually disjoint. Thus $\tilde{Y}_{k_{0}}^{k} \cup A_{k_{0}}^{k}$ is contained in $\tilde{X}_{k_{0}}$ for $k=1, \ldots, k_{0}+1$, and we regard them as subsets of $\left(M_{1}, g_{1}\right)$. Gluing disks to $\tilde{Y}_{k_{0}}^{k} \cup A_{k_{0}}^{k}\left(k=1, \ldots, k_{0}+1\right)$ and $\left(M_{1}, g_{1}\right) \backslash\left(\bigcup_{k=1}^{k_{0}+1} \tilde{Y}_{k_{0}}^{k}\right)$, we obtain compact conformally flat manifolds $M_{2}^{(k)}\left(k=1, \ldots, k_{0}+1\right)$ and $M^{\prime}$ such that

$$
M_{1}=M_{2}^{(1)} \sharp \ldots \sharp M_{2}^{\left(k_{0}+1\right)} \sharp M^{\prime}
$$

and $\pi_{1}\left(M_{2}^{(k)}\right) \cong \pi_{1}\left(M_{2}\right)$ as in (3.0.1). Then, by van Kampen's theorem and the Gruško-Neumann theorem, $d\left(M_{1}\right)=\left(k_{0}+1\right) d\left(M_{2}\right)+d\left(M^{\prime}\right)$ holds. This contradicts our definition of $k_{0}$. Thus there exists $k^{\prime}\left(0 \leq k^{\prime} \leq k_{0}\right)$ such that either (1) or (2) holds.

(3.k.1): $\varphi\left(A_{k}^{j} \cup \tilde{Y}_{k}^{j}\right) \subset X$ for $j=0, \ldots, k-1, \varphi\left(A_{k}^{k}\right) \subset X$ and $\varphi\left(\tilde{Y}_{k}^{k}\right) \supset \hat{Y}$. Define $T$ by

$$
T=\left(M, \hat{g}\left(l^{\prime}\right)\right) \backslash \bigcup_{j=0}^{k} \tilde{Y}_{k}^{j}=\tilde{X}_{k} \cup A_{k} \backslash \bigcup_{j=1}^{k} \tilde{Y}_{k}^{j} .
$$

Since $\varphi\left(\tilde{Y}_{k}^{j} \cup A_{k}^{j}\right) \subset X$ for $j=0, \ldots, k-1, \tilde{Y}_{k}^{j} \cup A_{k}^{j}(j=0, \ldots, k)$ are mutually disjoint as in (3.1.3). Then, clearly, $T$ is connected and $T$ contains $A_{k}^{j}$ for $j=$ $0, \ldots, k$ (see Figure 3 ). Since $\varphi\left(\tilde{Y}_{k}^{j}\right)$ is contained in $X$ for $j=0, \ldots, k-1$ and 
$\varphi\left(\tilde{Y}_{k}^{k}\right)$ contains $\hat{Y}$, we obtain

$$
\varphi(T)=\left(M, \hat{g}_{l}\right) \backslash \bigcup_{j=0}^{k} \varphi\left(\tilde{Y}_{k}^{j}\right) \subset X \backslash \bigcup_{j=0}^{k-1} \varphi\left(\tilde{Y}_{k}^{j}\right),
$$

and hence $i_{X} \circ \varphi$ is defined on $T$. Moreover, since

$$
i_{X} \circ \varphi(T) \subset i_{X}(X) \backslash\left(\bigcup_{j=0}^{k-1} i_{X} \circ \varphi\left(\tilde{Y}_{k}^{j}\right)\right) \subset \tilde{X}_{k} \backslash \bigcup_{j=1}^{k} \tilde{Y}_{k}^{j},
$$

$i_{X} \circ \varphi$ defines a conformal embedding $i_{X} \circ \varphi: T \longrightarrow T$. We denote this conformal embedding by $\xi$. Define $\hat{T}$ by

$$
\hat{T}=T \backslash \bigcup_{j=0}^{k} A_{k}^{j}
$$

Then

$$
\varphi(\hat{T})=\left(M, \hat{g}_{l}\right) \backslash \bigcup_{j=0}^{k} \varphi\left(\tilde{Y}_{k}^{j} \cup A_{k}^{j}\right) \subset X \backslash \bigcup_{j=0}^{k-1} \varphi\left(\tilde{Y}_{k}^{j} \cup A_{k}^{j}\right) .
$$

Thus we have

$$
\xi(\hat{T})=i_{X} \circ \varphi(\hat{T}) \subset \tilde{X}_{k} \backslash \bigcup_{j=0}^{k-1} i_{X} \circ \varphi\left(\tilde{Y}_{k}^{j} \cup A_{k}^{j}\right)=\hat{T} .
$$

Since $A_{k}^{k}$ is contained in $T, \xi^{j}\left(A_{k}^{k}\right)$ is defined for $j \geq 0$ and $\xi^{j}\left(A_{k}^{k}\right)=A_{k}^{k+j}$ is contained in $T$. Clearly $T \backslash \xi\left(A_{k}^{k}\right)$ has at least two connected components. Since $A_{k}^{j}$ $(j=0, \ldots, k)$ are mutually disjoint, so are $A_{k}^{j+1}=\xi\left(A_{k}^{j}\right)(j=0, \ldots, k)$. In particular, $A_{k}^{k+1}$ and $A_{k}^{j}(j=1, \ldots, k)$ are mutually disjoint. Since $\varphi\left(A_{k}^{k}\right)$ is contained in $X, \xi\left(A_{k}^{k}\right)=i_{X} \circ \varphi\left(A_{k}^{k}\right)$ is contained in $i_{X}(X)$ and hence $A_{k}^{k+1}$ and $A_{k}$ are disjoint. This shows that

$$
A_{k}^{k+1} \subset T \backslash \bigcup_{j=0}^{k} A_{k}^{j}=\hat{T} .
$$

Thus $\xi^{j}\left(A_{k}^{k+1}\right) \subset \xi^{j}(\hat{T}) \subset \hat{T}$ holds for $j \geq 0$. Note that $\hat{T}$ is a closed set and hence $\hat{T}$ contains the closure of $\xi^{j}\left(A_{k}^{k+1}\right)$ for $j \geq 0$. Then it is easy to see that $T \backslash \xi^{j}\left(A_{k}^{k+1}\right)$ has exactly two connected components. The set $T \backslash A_{k}^{k+1}$ is described as follows: Since $\left(M, \hat{g}\left(l^{\prime}\right)\right) \backslash A_{k}^{k}$ has two connected components $\tilde{Y}_{k}^{k}$ and $\left(M, \hat{g}\left(l^{\prime}\right)\right) \backslash\left(\tilde{Y}_{k}^{k} \cup A_{k}^{k}\right)$, $\left(M, \hat{g}_{l}\right) \backslash \varphi\left(A_{k}^{k}\right)$ has two connected components $\left(M, \hat{g}_{l}\right) \backslash \varphi\left(\tilde{Y}_{k}^{k} \cup A_{k}^{k}\right)$ and $\varphi\left(\tilde{Y}_{k}^{k}\right)$. Note that $\left(M, \hat{g}_{l}\right) \backslash \varphi\left(\tilde{Y}_{k}^{k} \cup A_{k}^{k}\right)$ is contained in $X$, since $\varphi\left(\tilde{Y}_{k}^{k}\right) \supset \hat{Y}$ and $\varphi\left(A_{k}^{k}\right) \subset X$. Then two connected components of $\left(M, \hat{g}\left(l^{\prime}\right)\right) \backslash A_{k}^{k+1}$, say $L$ and $N$, satisfy $L=$ $i_{X}\left(\left(M, \hat{g}_{l}\right) \backslash \varphi\left(\tilde{Y}_{k}^{k} \cup A_{k}^{k}\right)\right) \subset \tilde{X}_{k}$ and $N \supset A_{k} \cup \tilde{Y}_{k}$. Since $\tilde{Y}_{k}^{j} \cup A_{k}^{j}(j=0, \ldots, k)$ are mutually disjoint,

$$
L \supset \bigcup_{j=0}^{k-1} i_{X} \circ \varphi\left(\tilde{Y}_{k}^{j} \cup A_{k}^{j}\right)=\bigcup_{j=1}^{k}\left(\tilde{Y}_{k}^{j} \cup A_{k}^{j}\right) .
$$


Denote two connected components of $T \backslash A_{k}^{k+1}$ by $T_{0}$ and $T_{0}^{\prime}$ so that $T_{0}=T \cap L$ and $T_{0}^{\prime}=T \cap N$. Note that $T_{0}^{\prime}$ contains $A_{k}$. Since $L \cup A_{k}^{k+1}=i_{X}\left(\left(M, \hat{g}_{l}\right) \backslash \varphi\left(\tilde{Y}_{k}^{k}\right)\right)$,

$$
\begin{aligned}
\xi(T) & =i_{X}\left(\left(M, \hat{g}_{l}\right) \backslash \bigcup_{j=0}^{k} \varphi\left(\tilde{Y}_{k}^{j}\right)\right) \\
& =\left(L \cup A_{k}^{k+1}\right) \backslash \bigcup_{j=1}^{k} \tilde{Y}_{k}^{j} \\
& =\left(L \cup A_{k}^{k+1}\right) \cap\left(\left(M, \hat{g}_{l}\right) \backslash \bigcup_{j=0}^{k} \tilde{Y}_{k}^{j}\right) .
\end{aligned}
$$

Since $L \cap A_{k}^{k+1}$ is contained in $\tilde{X}_{k}$, we see that

$$
\begin{aligned}
\xi(T) & =\left(L \cup A_{k}^{k+1}\right) \cap T \\
& =T_{0} \cup A_{k}^{k+1} .
\end{aligned}
$$

In particular, $T_{0} \supset \xi(\hat{T})$ and $T_{0}^{\prime}=T \backslash \xi(T)$ hold. Denote two connected components of $T \backslash \xi^{j}\left(A_{k}^{k+1}\right)$ by $T_{j}$ and $T_{j}^{\prime}$ so that $T_{j} \supset \xi^{j}(T)$ and $T_{j}^{\prime} \supset \xi^{j}\left(T_{0}^{\prime}\right)$. Then we see that

$$
\begin{aligned}
& T_{j} \cup \xi^{j}\left(A_{k}^{k+1}\right) \supset \xi^{j+1}(T), \\
& T_{j} \supset \xi^{j+1}(\hat{T}), \\
& T_{j}^{\prime} \supset A_{k}^{j}, \\
& T_{j}^{\prime} \supset \xi^{j}\left(T_{0}^{\prime}\right)=\xi^{j}(T) \backslash \xi^{j+1}(T)
\end{aligned}
$$

hold. Take $\xi^{i}\left(A_{k}^{k+1}\right)=\xi^{k+1}\left(A_{k}^{i}\right)$ and $\xi^{j}\left(A_{k}^{k+1}\right)=\xi^{k+1}\left(A_{k}^{j}\right)$ for $0 \leq i<j \leq k$. Since $A_{k}^{j}$ and $A_{k}^{i}$ are disjoint, so are $\xi^{k+1}\left(A_{k}^{j}\right)$ and $\xi^{k+1}\left(A_{k}^{i}\right)$. Since $\xi^{j}\left(A_{k}^{k+1}\right) \subset \xi^{j}(\hat{T}) \subset$ $\xi^{i+1}(\hat{T}) \subset T_{i}$, either $T_{j} \cup \xi^{j}\left(A_{k}^{k+1}\right) \subset T_{i}$ or $T_{j}^{\prime} \cup \xi^{j}\left(A_{k}^{k+1}\right) \subset T_{i}$ holds. Assume $T_{j} \cup \xi^{j}\left(A_{k}^{k+1}\right) \subset T_{i}$. Then $T_{j}^{\prime} \supset T_{i}^{\prime} \cup \xi^{i}\left(A_{k}^{k+1}\right)$. But since $\xi^{i}\left(A_{k}^{k+1}\right)=\xi^{j}\left(A_{k}^{k+i-j+1}\right)$ and $1 \leq k+i-j+1 \leq k, A_{k}^{k+i-j+1}=\xi\left(A_{k}^{k+i-j}\right)$ is contained in $\xi(T)$. This shows $\xi^{i}\left(A_{k}^{k+1}\right) \subset \xi^{j} \circ \xi(T)=\xi^{j}\left(T_{0} \cup A_{k}^{k+1}\right) \subset\left(T_{j} \cup \xi^{j}\left(A_{k}^{k+1}\right)\right)$. A contradiction. Thus $T_{j}^{\prime} \cup \xi^{j}\left(A_{k}^{k+1}\right)$ is contained in $T_{i}$. In particular, we have

$$
\begin{cases}\left(T_{i}^{\prime} \cup \xi^{i}\left(A_{k}^{k+1}\right)\right) \cap\left(T_{j}^{\prime} \cup \xi^{j}\left(A_{k}^{k+1}\right)\right)=\emptyset & \text { if } i \neq j, \\ T_{i}^{\prime} \supset A_{k}^{j} & \text { if } i=j .\end{cases}
$$

Since $\xi^{k+1}(T) \subset \xi^{j+1}(T) \subset T_{j} \cup \xi^{j}\left(A_{k}^{k+1}\right)$ holds for $j=0, \ldots, k, \bigcup_{j=0}^{k} T_{j}^{\prime}$ is contained in $Y \backslash \xi^{k+1}(T)$. On the other hand, since $T_{j}^{\prime}$ contains $\xi^{j}(T) \backslash \xi^{j+1}(T)$ and $T \backslash \xi^{k+1}(T)=\bigcup_{j=0}^{k}\left(\xi^{j}(T) \backslash \xi^{j+1}(T)\right), T \backslash \xi^{k+1}(T)$ is contained in $\bigcup_{j=0}^{k} T_{j}^{\prime}$. Thus, $T \backslash \xi^{k+1}(T)=\bigcup_{j=0}^{k} T_{j}^{\prime}$. Identifying $S\left(\alpha_{0}\right)$ defined in (3.0.1) with each $A_{k}^{j}$, $j=1, \ldots k$, we can glue $E\left(\alpha_{0}\right) \cup S\left(\alpha_{0}\right)$ to $T$ and obtain a compact, connected and conformally flat manifold $M^{\prime}$ as in (3.0.1). Denote $E\left(\alpha_{0}\right) \cup S\left(\alpha_{0}\right)$ glued to $A_{k}^{j}$ by $D_{j}$ (see Figure 4). By the discussion above, we see that

$$
\left\{\begin{array}{l}
M^{\prime} \backslash \xi^{k+1}(\hat{T})=\bigcup_{j=0}^{k}\left(\xi^{k+1}\left(A_{k}^{j}\right) \cup T_{j}^{\prime} \cup D_{j}\right), \\
\left(\xi^{k+1}\left(A_{k}^{j}\right) \cup T_{j}^{\prime} \cup D_{j}\right) \cap\left(\xi^{k+1}\left(A_{k}^{i}\right) \cup T_{i}^{\prime} \cup D_{i}\right)=\emptyset \quad \text { if } i \neq j .
\end{array}\right.
$$


Define $D_{j, m}$ for $j=0, \ldots, k$ and $m=0, \ldots, 3$ by $D_{j, 0}=D_{j} \backslash A_{k}^{j}, D_{j, 1}=D_{j}, D_{j, 2}=$ $T_{j}^{\prime} \cup D_{j}$ and $D_{j, 3}=\xi^{k+1}(T) \cup D_{j, 2}$. Then we see that $D_{j, 0} \subset D_{j, 1} \subset D_{j, 2} \subset D_{j, 3}$ and

$$
\begin{aligned}
& M^{\prime} \backslash T=\bigcup_{j=0}^{k} D_{j, 0}, \\
& M^{\prime} \backslash \hat{T}=\bigcup_{j=0}^{k} D_{j, 1}, \\
& M^{\prime} \backslash \xi^{k+1}(T)=\bigcup_{j=0}^{k} D_{j, 2}, \\
& M^{\prime} \backslash \xi^{k+1}(\hat{T})=\bigcup_{j=0}^{k} D_{j, 3} .
\end{aligned}
$$

Applying the same argument as in (3.0.1) to each $j$, we see that $\xi^{k+1}$ is extended to a conformal transformation $\eta$ on $M^{\prime}$. Since $\eta\left(D_{j, 0}\right)=D_{j, 2}$ and $\eta\left(D_{j, 1}\right)=D_{j, 3}$ by our construction, $\eta$ is an essential conformal transformation on $M^{\prime}$ as we have seen in (3.0.1). Therefore $M^{\prime}$ is conformal to the standard $n$-sphere $S^{n}(1)$. Note that $D_{j, m}$ is a closed round $n$-disk if $m=0,2$ and an open round $n$-disk if $m=1,3$. Thus, for some $\beta_{0}>0$, we may regard $M^{\prime} \backslash\left(D_{0,1} \cup D_{1,1}\right)$ as the closure of $S\left(\beta_{0}\right)$ defined in the same way as $S\left(\alpha_{0}\right)$ in (3.0.1). Since $\eta\left(M^{\prime} \backslash\left(D_{0,1} \cup D_{1,1}\right)\right)=M^{\prime} \backslash\left(D_{0,3} \cup D_{1,3}\right)$, and $D_{j, 3} \backslash \xi^{k+1}\left(A_{k}^{j}\right) \supset D_{j, 1}$, there exists $\beta_{0}^{\prime}<\beta_{0}$ such that $D_{0,3} \supset E\left(-\beta_{0}^{\prime}\right) \supset$ (the closure of $\left.D_{0,1}\right)=E\left(-\beta_{0}\right)$ and $D_{1,3} \supset E\left(\beta_{0}^{\prime}\right) \supset\left(\right.$ the closure of $\left.D_{1,1}\right)=E\left(\beta_{0}\right)$, that is, $\eta\left(M^{\prime} \backslash\left(D_{0,1} \cup D_{1,1}\right)\right)=\eta\left(S\left(\beta_{0}\right)\right)$ is contained in $S\left(\beta_{0}^{\prime}\right)$. But by Lemma 4.13 in the next section, such an $\eta$ does not exist. Thus case (1) of (3.1.3) does not occur if $k \geq 1$. See Figure 4 .

(3.k.2): $\varphi\left(A_{k}^{k}\right) \subset Y$. Since $\left(M, \hat{g}\left(l^{\prime}\right)\right) \backslash A_{k}^{k}$ has two connected components $\left(M, \hat{g}\left(l^{\prime}\right)\right) \backslash\left(\tilde{Y}_{k}^{k} \cup A_{k}^{k}\right)$ and $\tilde{Y}_{k}^{k}$, either $\varphi\left(\tilde{Y}_{k}^{k}\right)$ or $\varphi\left(\left(M, \hat{g}\left(l^{\prime}\right)\right) \backslash\left(A_{k}^{k} \cup \tilde{Y}_{k}^{k}\right)\right)$ is contained in $Y$. If $\varphi\left(\tilde{Y}_{k}^{k}\right)$ is contained in $Y$, we have a conformal embedding $\varphi \circ\left(i_{X} \circ \varphi\right)^{k}$ : $\tilde{Y}^{k} \longrightarrow Y$. If $\varphi\left(\left(M, \hat{g}\left(l^{\prime}\right)\right) \backslash\left(\tilde{Y}_{k}^{k} \cup A_{k}^{k}\right)\right)$ is contained in $Y$, then $\varphi\left(\tilde{Y}_{k}\right)$ is contained in $Y$, since $A_{k}^{k} \cup \tilde{Y}_{k}^{k} \subset \tilde{X}_{k}$. We can derive a contradiction in each case as in (3.0.1).

By the above discussion, we conclude that there is no conformal diffeomorphism between $\left(M, \hat{g}_{l}\right)$ and $\left(M, \hat{g}\left(l^{\prime}\right)\right)$. This completes the proof of Theorem 3.1.

Recall that Lemma 3.3 is also true for noncompact manifolds. Actually, a part of the proof of Theorem 3.1 also works for noncompact manifolds. Let $\left(M_{1}, g_{1}\right)$ and $\left(M_{2}, g_{2}\right)$ be conformally flat manifolds, possibly noncompact, which are not diffeomorphic to $S^{n}$ nor $\mathbf{R}^{n}$. Let $M=M_{1} \sharp M_{2}$ and $\left(M, \hat{g}_{l}\right)$ be as above. We do not assume that $d\left(M_{1}\right) \geq d\left(M_{2}\right)$ here, and we define $\left(M, \hat{g}\left(l^{\prime}\right)\right)$ by setting $k_{0}=0$ (so $\left(M, \hat{g}\left(l^{\prime}\right)\right)$ contains a subset isometric to $\left.\left(0,2 l+l^{\prime}\right) \times S^{n-1}(1)\right)$. Other notations are the same as above.

Then we have two conformally flat Riemannian manifolds, and there is a diffeomorphism $\varphi_{0}$ between them which maps $i_{X}(\hat{X})\left(\right.$ resp. $\left.i_{Y}(\hat{Y})\right) \subset\left(M, \hat{g}\left(l^{\prime}\right)\right)$ to $\hat{X}$ (resp. $\hat{Y})$.

Theorem 3.4. If $l^{\prime}>C(n, l)$, where $C(n, l)$ is the constant in Lemma 3.3, then there is no conformal diffeomorphism between $\left(M, \hat{g}\left(l^{\prime}\right)\right)$ and $\left(M, \hat{g}_{l}\right)$ isotopic to $\varphi_{0}$. 

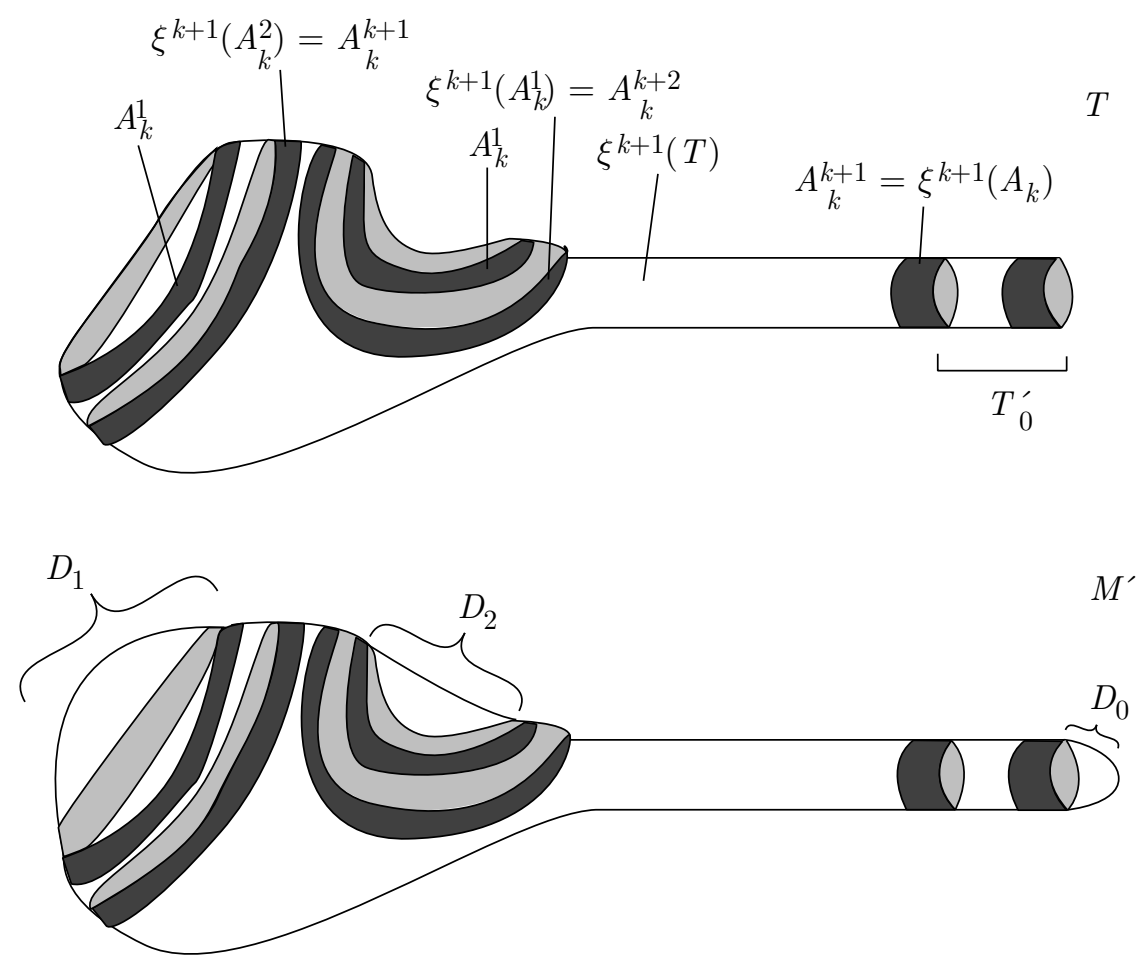

FiguRE 4

In other words, $\hat{g}_{l}$ and $\hat{g}\left(l^{\prime}\right)$ represent distinct elements in the Teichmüller space of flat conformal structures on $M$.

Proof. Assume that there is a conformal diffeomorphism $\varphi:\left(M, \hat{g}\left(l^{\prime}\right)\right) \longrightarrow\left(M, \hat{g}_{l}\right)$, which is isotopic to $\varphi_{0}$. By Lemma 3.3, there is a subset $A_{0}$ of $S_{0}$ such that $\varphi\left(A_{0}\right)$ is contained in either $X$ or $Y$. We may assume $\varphi\left(A_{0}\right) \subset X$ by interchanging $M_{1}$ and $M_{2}$, if necessary. Since $\varphi$ is isotopic to $\varphi_{0}, \varphi\left(\tilde{X}_{0}\right)$ is contained in $X$. Then $i_{X} \circ \varphi$ is defined on $\tilde{X}_{0} \cup A_{0}$ and $i_{X} \circ \varphi\left(A_{0} \cup \tilde{X}_{0}\right) \subset \tilde{X}$. Since $\varphi \circ \varphi_{0}^{-1}$ is isotopic to the identity map, it is easy to see that $X \backslash \varphi\left(\tilde{X}_{0} \cup A_{0}\right)$ must be compact. Therefore, $\tilde{X}_{0} \backslash i_{X} \circ \varphi\left(\tilde{X}_{0} \cup A_{0}\right)$ is also compact. As in (3.0.1), we can regard $\tilde{X}_{0} \cup A_{0}$ as a subset of $\left(M_{1}, g_{1}\right)$. Through this, define $D_{0}, D_{1}, D_{2}$ and $D_{3}$ as in (3.0.1). Let $M_{1}^{\prime}$ and $M_{2}^{\prime}$ be as in (3.0.1). Then, since $\tilde{X}_{0} \backslash i_{X} \circ \varphi\left(\tilde{X}_{0} \cup A_{0}\right)$ is compact, $M_{2}^{\prime}$ is compact and simply connected by the same reasoning as in (3.0.1). Hence $M_{2}^{\prime}$ is conformally equivalent to $S^{n}$. Thus we can extend the conformal diffeomorphism $i_{X} \circ \varphi$ : $\tilde{X}_{0} \cup A_{0} \longrightarrow i_{X} \circ \varphi\left(\tilde{X}_{0} \cup A_{0}\right)$ to a global conformal transformation $\eta$ on $\left(M_{1}, g_{1}\right)$, and $\eta$ is an essential conformal transformation as in (3.0.1). If $M_{1}$ is compact, then $M_{1}$ must be diffeomorphic to $S^{n}$, and this contradicts our assumption. Therefore, $M_{1}$ is noncompact. Let $\left(\tilde{M}_{1}, \tilde{g}_{1}\right)$ be the Riemannian universal covering of $\left(M_{1}, g_{1}\right)$. Fix a lift $\tilde{D}_{i}$ of $D_{i}, i=0,1,2$ and 3 , so that $\tilde{D}_{i} \subset \tilde{D}_{j}$ for $i<j$. Then there is a conformal transformation $\tilde{\eta}$ on $\left(\tilde{M}_{1}, \tilde{g}_{1}\right)$, which is a lift of $\eta$, such that $\tilde{\eta}\left(\tilde{D}_{1}\right)=\tilde{D}_{3}$. Fix a developing map $\Phi$ of $\left(\tilde{M}_{1}, \tilde{g}_{1}\right)$. Since the $\tilde{D}_{i}$ 's are conformally equivalent to a round ball of $S^{n}, \Phi$ is injective on $\tilde{D}_{3}$. Then, it is clear that the conformal 
transformation $\xi$ of $S^{n}$ satisfying $\Psi \circ \tilde{\eta}=\xi \circ \Psi$ is a loxodromic transformation of $S^{n}$ whose repulsive fixed point $q_{1}$ is in $\Psi\left(\tilde{D}_{3}\right)$. Since $\Psi \circ \tilde{\eta}^{k}=\xi^{k} \circ \Psi,\left(\tilde{M}_{1}, \tilde{g}_{1}\right)$ contains a subset conformally equivalent to $\mathbf{R}^{n} \cong S^{n} \backslash q_{2}$, where $q_{2}$ is the attractive fixed point of $\xi$. If this set $U$ is a proper subset of $\tilde{M}_{1}$, then the boundary of $U$ is mapped locally homeomorphically onto a point $q_{2}$ by $\Psi$. Therefore, $\tilde{M}_{1}$ is diffeomorphic to $S^{n}$. But here, since we assume $M_{1}$ to be noncompact, $\left(\tilde{M}_{1}, \tilde{g}_{1}\right)$ must be conformally equivalent to $\mathbf{R}^{n}$. On the other hand, the set $U$ can be considered as a lift of the limit of $\eta^{k}\left(D_{1}\right)$ as $k \rightarrow \infty$, thus $\left(M_{1}, g_{1}\right)$ itself must be conformal to $\mathbf{R}^{n}$. A contradiction. This completes the proof.

Remark. If $M_{1}$ or $M_{2}$ is diffeomorphic to either $S^{n}$ or $\mathbf{R}^{n}$, then $\left(M, \hat{g}_{l}\right)$ and $\left(M, \hat{g}\left(l^{\prime}\right)\right)$ may be conformally equivalent. This can be seen as in Remark 2 following Theorem 3.1 .

The same argument which shows Corollary 3.2 leads to the following corollary.

Corollary 3.5. Let $M=M_{1} \sharp M_{2}$, where $M_{1}$ and $M_{2}$ are conformally flat, possibly noncompact, manifolds and each of them is diffeomorphic to neither $S^{n}$ nor $\mathbf{R}^{n}$. Then the Teichmüller space of flat conformal structures on $M$ is noncompact.

\section{Proof of LemMas}

To prove Lemma 3.3, we need some basic facts on a conformal invariant called the modulus, which is related to the theory of quasi-conformal mappings (see [21] for a detailed exposition on this subject). Let $(M, g)$ be a Riemannian manifold and $\Delta$ an interval. A continuous map $\gamma: \Delta \longrightarrow M$ is called a path. A path $\gamma$ is called a closed path if $\Delta$ is a closed interval. If $\Delta^{\prime}$ is a subinterval of $\Delta,\left.\gamma\right|_{\Delta^{\prime}}$ is called a subpath of $\gamma$.

Definition 4.1. Let $\Delta=[a, b]$ and $A=\left\{a_{0}<a_{1}<\cdots<a_{j+1} ; a_{0}=a, a_{j+1}=b\right\}$ a subdivision of $\Delta$. Denote the distance between $p$ and $q$ with respect to a metric $g$ by $\operatorname{dist}_{g}(p, q)$. The length $l(\gamma)$ of $\gamma$ is defined by

$$
l(\gamma)=\sup _{A} \sum_{i=0}^{j} \operatorname{dist}_{g}\left(\gamma\left(a_{i}\right), \gamma\left(a_{i+1}\right)\right),
$$

where the supremum is taken over all subdivisions of $\Delta$.

Definition 4.2. A path $\gamma$ is said to be rectifiable if the length of $\gamma$ is finite. And a path $\gamma$ is called locally rectifiable if every closed subpath of $\gamma$ is rectifiable.

If $\gamma$ is rectifiable, then $\gamma$ is parametrized by arclength. In what follows, we assume that a rectifiable path is parametrized by arclength, and denote this parameter by $s$. Then, for a rectifiable path $\gamma$, the line integral along $\gamma$ makes sense. These notions are also defined for a locally rectifiable path in a natural way. Let $\Gamma$ be a path family.

Definition 4.3. A Borel function $\rho: M \longrightarrow[0, \infty]$ is called an admissible function of $\Gamma$, if, for any locally rectifiable path $\gamma$ contained in $\Gamma, \rho$ satisfies

$$
\int_{\gamma} \rho d s \geq 1 .
$$

The set of all admissible functions of $\Gamma$ is denoted by $\mathcal{F}(\Gamma)$. 
Definition 4.4. For a path family $\Gamma$, the modulus $\mathcal{M}(\Gamma)$ is defined by

$$
\mathcal{M}(\Gamma)=\inf _{\rho \in \mathcal{F}(\Gamma)} \int_{M} \rho^{n} d V_{g},
$$

where $d V_{g}$ denotes the volume element of $g$.

Let $E, F$ and $G$ be Borel sets of $(M, g)$. Define $\Delta(E, F ; G)$ by

$\Delta(E, F ; G)=\{$ closed non-constant paths $\gamma ; \gamma(0) \in E$,

$$
\gamma(l(\gamma)) \in F, \gamma(t) \in G \text { for } t \in(0, l(\gamma))\}
$$

The following are obvious consequences of the above definitions. For the proofs, see $[21]$.

Proposition 4.5. If $\Gamma$ is a subfamily of $\Gamma^{\prime}$, then $\mathcal{M}(\Gamma) \leq \mathcal{M}\left(\Gamma^{\prime}\right)$.

Proposition 4.6. If every path $\gamma$ contained in $\Gamma$ has a subpath belonging to $\Gamma^{\prime}$, then $\mathcal{M}(\Gamma) \leq \mathcal{M}\left(\Gamma^{\prime}\right)$.

\section{Proposition 4.7.}

$$
\mathcal{M}(\Delta(E, F ; G))=\inf _{\rho \in \mathcal{F}(\Delta(E, F ; G))} \int_{G} \rho^{n} d V_{g} .
$$

Proposition 4.8. Let $G$ be an open subset of a Riemannian manifold $(M, g)$ and $\Gamma=\Delta(E, F ; G)$ for subsets $E$ and $F$ of $G$. Assume that there exists a conformal embedding $\varphi$ of $\left(G,\left.g\right|_{G}\right)$ into a Riemannian manifold $(N, h)$. Then $\mathcal{M}(\Gamma)=\mathcal{M}(\varphi(\Gamma))$.

Proposition 4.8 asserts the conformal invariance of $\mathcal{M}(\Gamma)$. In the proof of Lemma 3.3 below, the above properties of the conformal invariant $\mathcal{M}(\Gamma)$ are used frequently.

Proof of Lemma 3.3. We use the same notations as in the proof of Theorem 3.1. Denote $X \cap Y$ by $S$. Then $S$ is isometric to $(0, l) \times S^{n-1}(1)$. If $\psi\left(\{0\} \times S^{n-1}(1)\right)$ is contained in $X$, then there exists $\delta>0$ such that $\psi\left((0, \delta) \times S^{n-1}(1)\right)$ is contained in $X$. Thus, there exist $\alpha$ and $\beta$ such that $[\alpha, \beta] \subset(0, \delta)$, and hence we get a subset $A$ of $S$ as in Lemma 3.3. Similarly, if one of $\psi\left(\{0\} \times S^{n-1}(1)\right) \subset Y$, $\psi\left(\left\{l^{\prime}\right\} \times S^{n-1}(1)\right) \subset X$ or $\psi\left(\left\{l^{\prime}\right\} \times S^{n-1}(1)\right) \subset Y$ holds, we see that $A$ in Lemma 3.3 exists. Therefore, to prove Lemma 3.3, it suffices to show that if we choose $l^{\prime}>C(n, l)$ and both $\psi\left(\{0\} \times S^{n-1}(1)\right) \cap \hat{X} \neq \emptyset$ and $\psi\left(\{0\} \times S^{n-1}(1)\right) \cap \hat{Y} \neq \emptyset$ hold, then either $\psi\left(\left\{l^{\prime}\right\} \times S^{n-1}(1)\right) \subset X$ or $\psi\left(\left\{l^{\prime}\right\} \times S^{n-1}(1)\right) \subset Y$ holds. Now assume that $\psi\left(\{0\} \times S^{n-1}(1)\right) \cap \hat{X} \neq \emptyset, \psi\left(\{0\} \times S^{n-1}(1)\right) \cap \hat{Y} \neq \emptyset, \psi\left(\left\{l^{\prime}\right\} \times S^{n-1}(1)\right) \cap \hat{X} \neq \emptyset$ and $\psi\left(\left\{l^{\prime}\right\} \times S^{n-1}(1)\right) \cap \hat{Y} \neq \emptyset$ hold. We prove Lemma 3.3 by deriving a contradiction in the case that $l^{\prime}$ is greater than a certain constant depending only on $n$ and $l$.

The set $S$ is conformally equivalent to a subset $S(a)$ of $S^{n}(1)$ defined as in the proof of Theorem 3.1 for some positive real number $a$. That is, there exists a conformal diffeomorphism $i: S \longrightarrow S(a)$. Note that $\{0\} \times S^{n-1}(1)$ and $\left\{l^{\prime}\right\} \times$ $S^{n-1}(1)$ are totally geodesic hypersurfaces of $\left(-\epsilon, l^{\prime}+\epsilon\right) \times S^{n-1}(1)$. Since $\psi$ and $i$ are conformal embeddings, a connected component $T_{0}$ of $i\left(\psi\left(\{0\} \times S^{n-1}(1)\right) \cap S\right)$ and a connected component $T_{l^{\prime}}$ of $i\left(\psi\left(\left\{l^{\prime}\right\} \times S^{n-1}(1)\right) \cap S\right)$ are intersections of round $(n-1)$-spheres of $S^{n}(1)$ and $S(a)$. Thus, there exist hyperplanes $V_{0}$ and $V_{l^{\prime}}$ of $\mathbf{R}^{n+1}$ such that $T_{0}=S(a) \cap V_{0}$ and $T_{l^{\prime}}=S(a) \cap V_{l^{\prime}}$. By our assumption, we may assume 


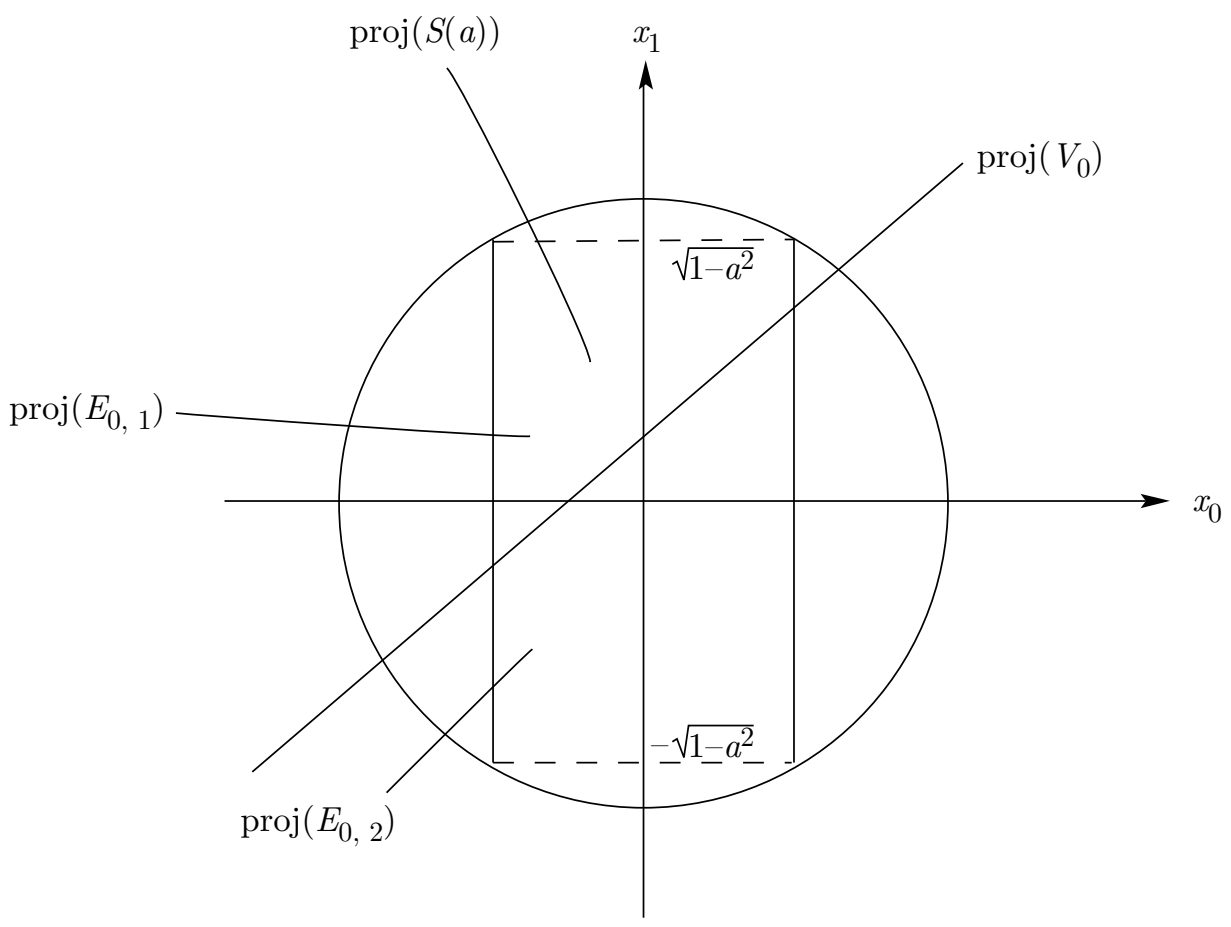

FigURE 5

that these hyperplanes satisfy

$$
\left\{\begin{array}{l}
V_{0} \cap\left\{x=\left(x_{0}, \ldots, x_{n}\right) \in S^{n}(1) \subset \mathbf{R}^{n+1} ; x_{0}=-a\right\} \neq \emptyset \\
V_{0} \cap\left\{x=\left(x_{0}, \ldots, x_{n}\right) \in S^{n}(1) \subset \mathbf{R}^{n+1} ; x_{0}=a\right\} \neq \emptyset \\
V_{l^{\prime}} \cap\left\{x=\left(x_{0}, \ldots, x_{n}\right) \in S^{n}(1) \subset \mathbf{R}^{n+1} ; x_{0}=-a\right\} \neq \emptyset \\
V_{l^{\prime}} \cap\left\{x=\left(x_{0}, \ldots, x_{n}\right) \in S^{n}(1) \subset \mathbf{R}^{n+1} ; x_{0}=a\right\} \neq \emptyset .
\end{array}\right.
$$

Denote two connected components of $S(a) \backslash T_{0}$ by $E_{0,1}$ and $E_{0,2}$ so that $E_{0,2}$ contains $T_{l^{\prime}}$. Similarly, denote two connected components of $S(a) \backslash T_{l^{\prime}}$ by $E_{l^{\prime}, 1}$ and $E_{l^{\prime}, 2}$ so that $E_{l^{\prime}, 2}$ contains $T_{0}$. Then $E_{0,2}$ contains $E_{l^{\prime}, 1}$, and $E_{l^{\prime}, 2}$ contains $E_{0,1}$. Let $v$ be a vector of $\mathbf{R}^{n+1}$ perpendicular to $V_{0}$. Then, by (4.9), the $x_{0}$-axis and $v$ are linearly independent. Denote the 2-dimensional subspace spanned by the $x_{0}$-axis and $v$ by $Q$. We may assume the $x_{1}$-axis is contained in $Q$. Let proj : $\mathbf{R}^{n+1} \longrightarrow Q$ be the orthogonal projection. Then $\operatorname{proj}\left(V_{0}\right)$ is a line in $Q$, and the $x_{1}$-coordinate of $\operatorname{proj}\left(V_{0}\right) \cap \operatorname{proj}(S(a))$ takes its value between $-\left(1-a^{2}\right)^{1 / 2}$ and $\left(1-a^{2}\right)^{1 / 2}$ (see Figure $5)$. Fix a positive real number $\epsilon_{0}$ so that $\epsilon_{0}<a$. We may assume $x=(0,1,0 \ldots, 0)$ is contained in $E_{0,1}$, and hence

$$
B_{1}=\left\{x=\left(x_{0}, \ldots, x_{n}\right) \in S(a) ; x_{1} \geq\left(1-\left(a-\epsilon_{0}\right)^{2}\right)^{1 / 2}\right\}
$$

is a round $n$-disk contained in $E_{0,1}$. We get a round $n$-disk $B_{2}$ contained in $E_{l^{\prime}, 1}$ in the same way. Define path families $\Gamma_{0}$ and $\Gamma$ by

$$
\begin{aligned}
& \Gamma_{0}=\Delta\left(\psi\left(\{0\} \times S^{n-1}(1)\right), \psi\left(\left\{l^{\prime}\right\} \times S^{n-1}(1)\right) ; \psi\left(\left(-\epsilon, l^{\prime}+\epsilon\right) \times S^{n-1}(1)\right)\right), \\
& \Gamma=\Delta\left(T_{0}, T_{l^{\prime}} ; S(a)\right) .
\end{aligned}
$$


For any $\gamma \in \Gamma, i^{-1}(\gamma)$ is a path between $\psi\left(\{0\} \times S^{n-1}(1)\right)$ and $\psi\left(\left\{l^{\prime}\right\} \times S^{n-1}(1)\right)$. Since $(M, g) \backslash \psi\left(\left(0, l^{\prime}\right) \times S^{n-1}(1)\right)$ has two connected components, there is a subpath of $i^{-1}(\gamma)$ contained in $\Gamma_{0}$. By Proposition 4.6 and Proposition 4.8, we see that

$$
\mathcal{M}\left(\Gamma_{0}\right) \geq \mathcal{M}\left(i^{-1}(\Gamma)\right)=\mathcal{M}(\Gamma) .
$$

Define a path family $\Gamma^{\prime}$ by

$$
\Gamma^{\prime}=\Delta\left(B_{1}, B_{2} ; S(a)\right)
$$

Then, by the definition of $B_{1}$ and $B_{2}$, every $\gamma^{\prime} \in \Gamma^{\prime}$ intersects $T_{0}$ and $T_{l^{\prime}}$. Thus there is a subpath of $\gamma^{\prime}$ which belongs to $\Gamma$. By Proposition 4.6,

$$
\mathcal{M}(\Gamma) \geq \mathcal{M}\left(\Gamma^{\prime}\right)
$$

Since the center of $B_{i}, i=1,2$, is contained in $S(0)=\left\{x=\left(x_{0}, \ldots x_{n}\right) \in S^{n}(1) \subset\right.$ $\left.\mathbf{R}^{n+1} ; x_{0}=0\right\}$, we can take the shortest geodesic $c$ between the centers of $B_{1}$ and $B_{2}$ so that $c$ is contained in $S(0)$. Let $p$ be the midpoint of $c$ and $q$ the antipodal point of $p$. Note that $q$ is contained in $S(0) \subset S(a)$. Define $\Gamma^{\prime \prime}$ by

$$
\Gamma^{\prime \prime}=\Delta\left(B_{1}, B_{2} ; S(a) \backslash\{q\}\right) .
$$

Since $\Gamma^{\prime \prime} \subset \Gamma^{\prime}, \mathcal{M}\left(\Gamma^{\prime}\right) \geq \mathcal{M}\left(\Gamma^{\prime \prime}\right)$ by Proposition 4.5. Let $\pi_{q}$ be the stereographic projection from $q$. By Proposition 4.8,

$$
\mathcal{M}\left(\Gamma^{\prime}\right) \geq \mathcal{M}\left(\Gamma^{\prime \prime}\right)=\mathcal{M}\left(\Delta\left(\pi_{q}\left(B_{1}\right), \pi_{q}\left(B_{2}\right) ; \pi_{q}(S(a) \backslash\{q\})\right) .\right.
$$

We may assume $\pi_{q}(c)$ corresponds to the $y_{1}$-axis of $\mathbf{R}^{n}=\pi_{q}\left(S^{n} \backslash\{q\}\right)$ and $\pi_{q}(S(0))$ corresponds to the $\left(y_{1}, \ldots, y_{n-1}\right)$-subspace. Let the Euclidean distance between two connected components of the boundaries of $\pi_{q}\left(S\left(a-\epsilon_{0}\right)\right)$ be $2 r_{0}$. Then the intersections of the $y_{n}$-axis and the boundaries of $\pi_{q}\left(S\left(a-\epsilon_{0}\right)\right)$ are $\left(0, \ldots, 0, \pm r_{0}\right)$, and $\pi_{q}\left(B_{1}\right)$ and $\pi_{q}\left(B_{2}\right)$ are disks centered at points of $y_{1}$-axis and each radius is greater than $r_{0}$. Set

$$
F=\left\{y=\left(y_{1}, \ldots, y_{n}\right) \in \mathbf{R}^{n} ; y_{2}^{2}+\cdots+y_{n}^{2}=r_{0}^{2}\right\} .
$$

Then, for some positive real numbers $h_{1}$ and $h_{2}$ with $h_{1}<h_{2}$,

$$
\begin{aligned}
& F \cap \partial\left(\pi_{q}\left(B_{1}\right)\right)=\left\{y=\left(y_{1}, \ldots, y_{n}\right) \in \mathbf{R}^{n} ; y_{2}^{2}+\cdots+y_{n}^{2}=r_{0}^{2}, y_{1}=h_{1}, h_{2}\right\}, \\
& F \cap \partial\left(\pi_{q}\left(B_{2}\right)\right)=\left\{y=\left(y_{1}, \ldots, y_{n}\right) \in \mathbf{R}^{n} ; y_{2}^{2}+\cdots+y_{n}^{2}=r_{0}^{2}, y_{1}=-h_{1},-h_{2}\right\},
\end{aligned}
$$

where $\partial$ denotes the boundary of a set. Define $F_{1}, F_{2}$ and $G$ by

$$
\begin{aligned}
& F_{1}=\left\{y=\left(y_{1}, \ldots, y_{n}\right) \in \mathbf{R}^{n} ; y_{2}^{2}+\cdots+y_{n}^{2} \leq r_{0}^{2}, y_{1}=h_{1}\right\}, \\
& F_{2}=\left\{y=\left(y_{1}, \ldots, y_{n}\right) \in \mathbf{R}^{n} ; y_{2}^{2}+\cdots+y_{n}^{2} \leq r_{0}^{2}, y_{1}=-h_{1}\right\}, \\
& G=\left\{y=\left(y_{1}, \ldots, y_{n}\right) \in \mathbf{R}^{n} ; y_{2}^{2}+\cdots+y_{n}^{2} \leq r_{0}^{2},\left|y_{1}\right| \leq\left|h_{1}\right|\right\} .
\end{aligned}
$$

Then, clearly, $F_{1} \subset \pi_{q}\left(B_{1}\right), F_{2} \subset \pi_{q}\left(B_{2}\right)$ and $G \subset \pi_{q}(S(a) \backslash\{q\})$ hold and hence $\Delta\left(F_{1}, F_{2} ; G\right)$ is contained in $\Delta\left(\pi_{q}\left(B_{1}\right), \pi_{q}\left(B_{2}\right) ; \pi_{q}(S(a) \backslash\{q\})\right)$. By our choices of $B_{1}, B_{2}$ and $p$, each of $\pi_{q}\left(B_{1}\right)$ and $\pi_{q}\left(B_{2}\right)$ is tangent to $\partial\left(\pi_{q}\left(S\left(a-\epsilon_{0}\right)\right)\right)$ at two points, and these two points are contained in the unit closed ball of $\mathbf{R}^{n}$, which is the image of the closure of the southern hemisphere by $\pi_{q}$ (see Figure 6 ). Since the Euclidean distance between these two tangent points is greater than $2 r_{0}$, we have $\left|h_{1}\right|<1$. Moreover, since $\mathcal{M}\left(\Delta\left(F_{1}, F_{2} ; G\right)\right)=\operatorname{Vol}_{n-1}\left(F_{1}\right)\left(2 h_{1}\right)^{1-n}$ (see for example $[21$, p. 52$])$, where $\operatorname{Vol}_{n-1}\left(F_{1}\right)$ denotes the $(n-1)$-dimensional Euclidean volume of $F_{1}$, we have

$$
\mathcal{M}\left(\Delta\left(\pi_{q}\left(B_{1}\right), \pi_{q}\left(B_{2}\right) ; \pi_{q}(S(a) \backslash\{q\})\right) \geq \operatorname{Vol}_{n-1}\left(F_{1}\right)\left(2 h_{1}\right)^{1-n}>2^{1-n} \operatorname{Vol}_{n-1}\left(F_{1}\right)\right.
$$




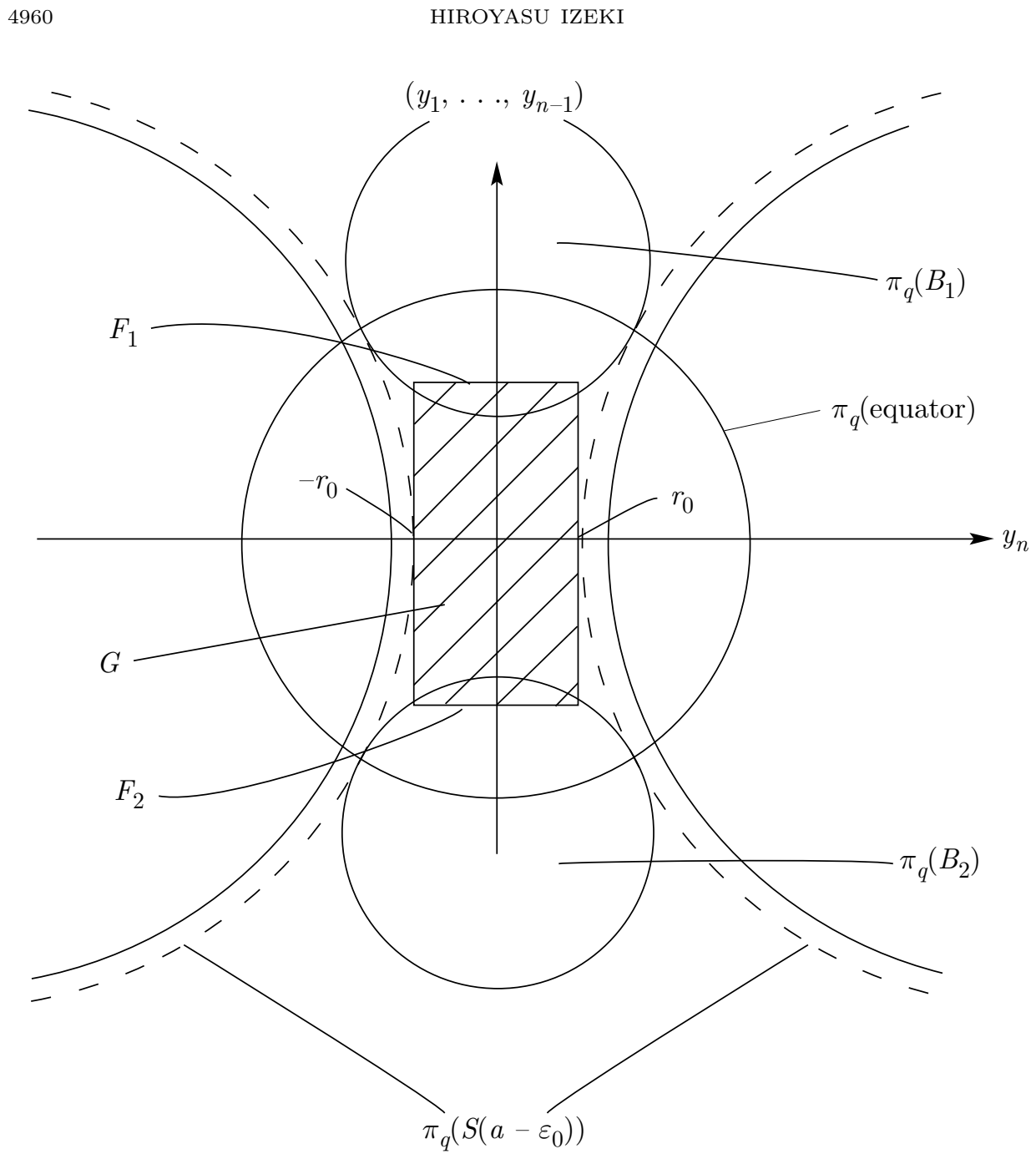

Figure 6

by Proposition 4.5. Combining with (4.10), (4.11) and (4.12), we have

$$
\mathcal{M}\left(\Gamma_{0}\right)>2^{1-n} \operatorname{Vol}_{n-1}\left(F_{1}\right)
$$

Since the radius $r_{0}$ of $F_{1}$ is determined by $a$ and $\epsilon_{0}$ and we can choose $\epsilon_{0}$ as, for instance, $a / 10, r_{0}$ is determined by $a$. Thus we may consider that $r_{0}$ is determined by $l$. Set $K(n, l)=2^{1-n} \operatorname{Vol}_{n-1}\left(F_{1}\right)$. For any $\gamma_{0} \in \psi^{-1}\left(\Gamma_{0}\right)=$ $\Delta\left(\{0\} \times S^{n-1}(1),\left\{l^{\prime}\right\} \times S^{n-1}(1) ;\left(-\epsilon, l^{\prime}+\epsilon\right) \times S^{n-1}(1)\right)$, clearly $l\left(\gamma_{0}\right) \geq l^{\prime}$ holds. Thus, for any $\gamma_{0} \in \psi^{-1}\left(\Gamma_{0}\right), f=l^{\prime-1}$ defined on $\left(-\epsilon, l^{\prime}+\epsilon\right) \times S^{n-1}(1)$ satisfies

$$
\int_{\gamma_{0}} f d s=l^{\prime-1} l\left(\gamma_{0}\right) \geq 1
$$

and hence $f \in \mathcal{F}\left(\psi^{-1}\left(\Gamma_{0}\right)\right)$. Define $\hat{\Gamma}_{0}$ by

$$
\hat{\Gamma}_{0}=\Delta\left(\{0\} \times S^{n-1}(1),\left\{l^{\prime}\right\} \times S^{n-1}(1) ;\left[0, l^{\prime}\right] \times S^{n-1}(1)\right) .
$$


Then, by Proposition 4.6, we have

$$
\mathcal{M}\left(\hat{\Gamma}_{0}\right) \geq \mathcal{M}\left(\psi^{-1}\left(\Gamma_{0}\right)\right)
$$

and clearly $\left.f\right|_{\left[0, l^{\prime}\right] \times S^{n-1}(1)}$ belongs to $\mathcal{F}\left(\hat{\Gamma}_{0}\right)$. Note that

$$
\begin{aligned}
\mathcal{M}\left(\hat{\Gamma}_{0}\right) & \leq \int_{\left[0, l^{\prime}\right] \times S^{n-1}(1)} f^{n} d s \\
& =l^{\prime-n} \operatorname{Vol}\left(S^{n-1}(1)\right) l^{\prime} \\
& =l^{\prime-n} \operatorname{Vol}\left(S^{n-1}(1)\right) .
\end{aligned}
$$

Define a positive constant $C(n, l)$ by

$$
C(n, l)=\left\{\operatorname{Vol}\left(S^{n-1}(1)\right) / K(n, l)\right\}^{1 / n-1}
$$

and choose $l^{\prime}$ so that $l^{\prime}$ is greater than $C(n, l)$. Then we have

$$
l^{\prime 1-n} \operatorname{Vol}\left(S^{n-1}(1)\right)<K(n, l)
$$

and hence

$$
\mathcal{M}\left(\psi^{-1}\left(\Gamma_{0}\right)\right) \leq \mathcal{M}\left(\hat{\Gamma}_{0}\right)<K(n, l)<\mathcal{M}\left(\Gamma_{0}\right) .
$$

This contradicts Proposition 4.8. This completes the proof of Lemma 3.3.

Using the modulus of path families, we can also prove the following lemma which is used in (3.k.1) of the previous section.

Lemma 4.13. There is no conformal transformation of $S^{n}(1)$ which sends the closure of $S\left(\beta_{0}\right)$ into $S\left(\beta_{0}^{\prime}\right)$ for $\beta_{0}^{\prime}<\beta_{0}$.

Proof. Assume that there exists a conformal transformation $\eta$ of $S^{n}(1)$ which satisfies $\eta\left(S\left(\beta_{0}\right)\right) \subset S\left(\beta_{0}^{\prime}\right)$ with $\beta_{0}^{\prime}<\beta_{0}$. We denote the interior of two connected components of $S^{n}(1) \backslash S\left(\beta_{0}\right)$ by $D_{0,1}$ and $D_{1,1}$ as in the previous section. Define two path families $\Gamma$ and $\Gamma^{\prime}$ by

$$
\begin{aligned}
& \Gamma=\Delta\left(\eta\left(\partial D_{0,1}\right), \eta\left(\partial D_{1,1}\right) ; \eta\left(S^{n} \backslash\left(D_{0,1} \cup D_{1,1}\right)\right)\right), \\
& \Gamma^{\prime}=\Delta\left(\partial E\left(-\beta_{0}^{\prime}\right), \partial E\left(\beta_{0}^{\prime}\right) ; \text { the closure of } S\left(\beta_{0}^{\prime}\right)\right) .
\end{aligned}
$$

By our assumption, every $\gamma^{\prime} \in \Gamma^{\prime}$ has a subpath belonging to $\Gamma$, and hence, by Proposition 4.6, $\mathcal{M}\left(\Gamma^{\prime}\right) \leq \mathcal{M}(\Gamma)$. Let $\pi_{p}$ be the stereographic projection from $p=(1,0, \ldots, 0) \in S^{n}(1) \subset \mathbf{R}^{n+1}$. An $(n-1)$-round sphere defined by $\{x=$ $\left(x_{0}, \ldots x_{n}\right) \in S^{n} \subset \mathbf{R}^{n+1} ; x_{0}$ is constant $\}$ is projected to a sphere of $\mathbf{R}^{n}$ centered at the origin by $\pi_{p}$. Thus, we have

$$
\begin{aligned}
& \pi_{p}\left(\partial D_{0,1}\right)=S^{n-1}\left(b_{1}\right) \subset \mathbf{R}^{n}=\pi_{p}\left(S^{n}(1) \backslash\{p\}\right), \\
& \pi_{p}\left(\partial D_{1,1}\right)=S^{n-1}\left(b_{2}\right), \\
& \pi_{p}\left(\partial E\left(-\beta_{0}^{\prime}\right)\right)=S^{n-1}\left(b_{1}^{\prime}\right), \\
& \pi_{p}\left(\partial E\left(\beta_{0}^{\prime}\right)\right)=S^{n-1}\left(b_{2}^{\prime}\right),
\end{aligned}
$$

with $b_{1}<b_{1}^{\prime}<b_{2}^{\prime}<b_{2}$. Denote the closed disk with radius $r$ of $\mathbf{R}^{n}$ by $D^{n}(r)$ and the interior of $D^{n}(r)$ by int $D^{n}(r)$. Since

$$
\begin{aligned}
\mathcal{M}\left(\Gamma^{\prime}\right) & =\mathcal{M}\left(\Delta\left(S^{n-1}\left(b_{1}^{\prime}\right), S^{n-1}\left(b_{2}^{\prime}\right) ; D^{n}\left(b_{2}^{\prime}\right) \backslash \operatorname{int} D^{n}\left(b_{1}^{\prime}\right)\right)\right) \\
& =\operatorname{Vol}\left(S^{n-1}(1)\right)\left\{\log \left(b_{2}^{\prime} / b_{1}^{\prime}\right)\right\}^{1-n}
\end{aligned}
$$


and

$$
\begin{aligned}
\mathcal{M}( & \Delta\left(\partial D_{0,1}, \partial D_{1,1} ; S^{n}(1) \backslash\left(D_{0,1} \cup D_{1,1}\right)\right) \\
\quad & =\mathcal{M}\left(S^{n-1}\left(b_{1}\right), S^{n-1}\left(b_{2}\right) ; D^{n}\left(b_{2}\right) \backslash \operatorname{int} D^{n}\left(b_{1}\right)\right) \\
\quad & =\operatorname{Vol}\left(S^{n-1}(1)\right)\left\{\log \left(b_{2} / b_{1}\right)\right\}^{1-n},
\end{aligned}
$$

by Proposition 4.8 and [21, p. 53], we have

$$
\mathcal{M}(\Gamma) \geq \mathcal{M}\left(\Gamma^{\prime}\right)>\mathcal{M}\left(\Delta\left(\partial D_{0,1}, \partial D_{1,1} ; S^{n}(1) \backslash\left(D_{0,1} \cup D_{1,1}\right)\right)\right)=\mathcal{M}\left(\eta^{-1}(\Gamma)\right) .
$$

Then it is clear that this contradicts Proposition 4.8. This completes the proof of Lemma 4.13.

\section{Appendix. The SPace of Flat COnformal structures on $\mathbf{R} P^{n} \sharp \mathbf{R} P^{n}$}

In this Appendix, we examine the space of flat conformal structures on $\mathbf{R} P^{n} \sharp \mathbf{R} P^{n}$, which is the simplest example of non- $C$-prime conformally flat manifolds, following the program mentioned in the introduction. Note that there exists a 2-fold covering $S^{1} \times S^{n-1} \longrightarrow \mathbf{R} P^{n} \sharp \mathbf{R} P^{n}$. Since any flat conformal structure on $S^{1} \times S^{n-1}$ is Kleinian (see [4] and [11]), for any flat conformal structure $C,\left(\mathbf{R} P^{n} \sharp \mathbf{R} P^{n}, C\right)$ is also Kleinian and conformal to the quotient of $S^{n} \backslash$ \{two points $\}$ by a discrete subgroup $\Gamma$ of the conformal transformation group of $S^{n}$, where $\Gamma$ is isomorphic to $\pi_{1}\left(\mathbf{R} P^{n} \sharp \mathbf{R} P^{n}\right) \cong \mathbf{Z} / 2 \mathbf{Z} * \mathbf{Z} / 2 \mathbf{Z}$. Then, by Theorem 2.7, $\left(\mathbf{R} P^{n} \sharp \mathbf{R} P^{n}, C\right)$ is conformal to some $\left(M_{1}, C_{1}\right) \sharp\left(M_{2}, C_{2}\right)$, where each $M_{i}$ is homeomorphic (diffeomorphic if $n \leq 6$ ) to $\mathbf{R} P^{n}$ and the $C_{i}$ 's are Kleinian structures on $M_{i}$. Since $\pi_{1}\left(M_{i}\right), i=1,2$, are finite, each $M_{i}$ must be diffeomorphic to $\mathbf{R} P^{n}$ by a theorem of Kuiper ([10]). Moreover each $C_{i}$ contains a constant curvature metric, that is, each $C_{i}$ is the standard (and hence unique) conformal structure on $\mathbf{R} P^{n}$. We denote this flat conformal structure by $C_{0}$. Then, for any $C,\left(\mathbf{R} P^{n} \sharp \mathbf{R} P^{n}, C\right)$ is conformal to some $\left(\mathbf{R} P^{n}, C_{0}\right) \sharp\left(\mathbf{R} P^{n}, C_{0}\right)$. And we see that deformations of flat conformal structures on $\mathbf{R} P^{n} \sharp \mathbf{R} P^{n}$ arise from varying $A, \psi_{1}$ and $\psi_{2}$ in Definition 2.6. Precisely speaking, for any $\left(\mathbf{R} P^{n} \sharp \mathbf{R} P^{n}, C\right)$, there exists $\left(\mathbf{R} P^{n} \sharp \mathbf{R} P^{n}, \hat{g}_{l}\right)$ constructed from two copies of $\left(\mathbf{R} P^{n}, g_{0}\right)$ as in section 3 such that $\hat{g}_{l}$ is contained in $C$, where $g_{0}$ denotes the metric of constant curvature 1 on $\mathbf{R} P^{n}$ (see below). In this case, $\left(\mathbf{R} P^{n} \sharp \mathbf{R} P^{n}, \hat{g}_{l}\right)$ is conformal to $\left(\mathbf{R} P^{n} \sharp \mathbf{R} P^{n}, \hat{g}_{l^{\prime}}\right)$ if and only if $l=l^{\prime}$. In particular, the space of flat conformal structures on $\mathbf{R} P^{n} \sharp \mathbf{R} P^{n}$ is parametrized by $l$ and hence 1-dimensional.

Since $S^{n} \backslash\{$ two points $\}$ is conformal to $\mathbf{R} \times S^{n-1}(1)$, for any flat conformal structure $C$ on $\mathbf{R} P^{n} \sharp \mathbf{R} P^{n},\left(\mathbf{R} P^{n} \sharp \mathbf{R} P^{n}, C\right)$ is conformal to $\mathbf{R} \times S^{n-1}(1) / \Gamma$, where $\Gamma \cong \mathbf{Z} / 2 \mathbf{Z} * \mathbf{Z} / 2 \mathbf{Z}$ is a discrete subgroup of the conformal transformation group $G$ of $\mathbf{R} \times S^{n-1}(1)$. Let $\gamma_{1}$ and $\gamma_{2}$ be the generators of $\Gamma$, each of which is of order 2. Note that $G$ coincides with the isometry group of $\mathbf{R} \times S^{n-1}(1)$ and, for any $\gamma \in G$, the action of $\gamma$ is written as $\gamma(r, p)=( \pm r+d, A p)$ for some real number $d$ and some $A \in O(n)$, where $(r, p)$ denotes a point of $\mathbf{R} \times S^{n-1}(1)$ with $r \in \mathbf{R}$ and $p \in S^{n-1}(1)$. Since $\gamma_{1}$ and $\gamma_{2}$ act freely on $\mathbf{R} \times S^{n-1}(1)$, we see easily that $\gamma_{1}$ and $\gamma_{2}$ are written as

$$
\left\{\begin{array}{l}
\gamma_{1}(r, p)=(-r+2 a, \bar{p}), \\
\gamma_{2}(r, p)=(-r+2 b, \bar{p}),
\end{array}\right.
$$


where $\bar{p}$ denotes the antipodal point of $p$. We may assume that $a$ is greater than $b$. Since $G$ coincides with the isometry group, $\mathbf{R} \times S^{n-1}(1) / \Gamma$ defines a Riemannian manifold, which is obtained as closing the boundaries of $[0, a-b] \times S^{n-1}(1)$ by the identification $p=\bar{p}$, where $p$ is a point of $\{0\} \times S^{n-1}(1)$ or $\{a-b\} \times S^{n-1}(1)$. We may regard this Riemannian manifold as $\left(\mathbf{R} P^{n} \sharp \mathbf{R} P^{n}, \hat{g}_{l}\right)$ constructed in section 3 with $l=a-b$. Let $C$ and $C^{\prime}$ be flat conformal structures on $\mathbf{R} P^{n} \sharp \mathbf{R} P^{n}$. Then there exists a discrete subgroup $\Gamma$ (resp. $\Gamma^{\prime}$ ) of $G$ such that the metric on $\mathbf{R} P^{n} \sharp \mathbf{R} P^{n}$ defined by $\mathbf{R} \times S^{n-1}(1) / \Gamma$ (resp. $\left.\mathbf{R} \times S^{n-1}(1) / \Gamma^{\prime}\right)$ is contained in $C$ (resp. $\left.\tilde{C}\right)$. Denote generators of $\Gamma$ and $\Gamma^{\prime}$ by $\gamma_{i}$ and $\gamma_{i}^{\prime}(i=1,2)$ respectively. As we have seen above, we may assume that $\gamma_{i}$ and $\gamma_{i}^{\prime}$ are written as

$$
\begin{gathered}
\left\{\begin{array}{l}
\gamma_{1}(r, p)=(-r+2 a, \bar{p}), \\
\gamma_{2}(r, p)=(-r+2 b, \bar{p}),
\end{array}\right. \\
\left\{\begin{array}{l}
\gamma_{1}^{\prime}(r, p)=\left(-r+2 a^{\prime}, \bar{p}\right), \\
\gamma_{2}^{\prime}(r, p)=\left(-r+2 b^{\prime}, \bar{p}\right),
\end{array}\right.
\end{gathered}
$$

for $a>b$ and $a^{\prime}>b^{\prime}$. Then $\mathbf{R} \times S^{n-1}(1) / \Gamma\left(\right.$ resp. $\left.\mathbf{R} \times S^{n-1}(1) / \Gamma^{\prime}\right)$ contains a subset isometric to $(0, a-b) \times S^{n-1}(1)\left(\right.$ resp. $\left.\left(0, a^{\prime}-b^{\prime}\right) \times S^{n-1}(1)\right)$. Suppose $C$ and $C^{\prime}$ are conformally equivalent. Then, since there exists $\gamma \in G$ such that $\Gamma=\gamma^{-1} \Gamma^{\prime} \gamma$ and $G$ is just the isometry group, $\mathbf{R} \times S^{n-1}(1) / \Gamma$ is isometric to $\mathbf{R} \times S^{n-1}(1) / \Gamma^{\prime}$ and hence $a-b=a^{\prime}-b^{\prime}$. Therefore the correspondence between a flat conformal structure $C$ and $a-b$ defines a map $F$ from the moduli space of flat conformal structures on $\mathbf{R} P^{n} \sharp \mathbf{R} P^{n}$ (i.e., the quotient space of the space of all flat conformal structures on $\mathbf{R} P^{n} \sharp \mathbf{R} P^{n}$ by the group of diffeomorphisms) to positive real numbers, and this map $F$ is clearly surjective. On the other hand, if $F(C)=F\left(C^{\prime}\right)$, then $\mathbf{R} \times S^{n-1}(1) / \Gamma=\left(\mathbf{R} P^{n} \sharp \mathbf{R} P^{n}, \hat{g}_{F(C)}\right)$ and $\mathbf{R} \times S^{n-1}(1) / \Gamma^{\prime}=\left(\mathbf{R} P^{n} \sharp \mathbf{R} P^{n}, \hat{g}_{F\left(C^{\prime}\right)}\right)$ are clearly isometric, and hence $C$ and $C^{\prime}$ are conformally equivalent. This shows that $F$ is injective and hence bijective. Moreover we have a representative $\hat{g}_{F(C)}$ of $C$. Therefore, as we mentioned in the previous paragraph, $\left(\mathbf{R} P^{n} \sharp \mathbf{R} P^{n}, \hat{g}_{l}\right)$ and $\left(\mathbf{R} P^{n} \sharp \mathbf{R} P^{n}, \hat{g}_{l^{\prime}}\right)$ constructed as above are conformally equivalent if and only if $l=l^{\prime}$. Since $\hat{g}_{l}$ depends continuously on $l$, we see that the map $F$ from the moduli space of flat conformal structures on $\mathbf{R} P^{n} \sharp \mathbf{R} P^{n}$ to positive real numbers is a homeomorphism. Thus $l$ defines a global coordinate of the moduli space.

In our construction of conformally flat metrics on a connected sum of conformally flat manifolds (in section 3), it seems that there are more parameters for deformations of flat conformal structures other than $l$ (for example, choices of points removed and isometries of a cylinder gluing $X$ and $Y$ ). But, for $\mathbf{R} P^{n} \sharp \mathbf{R} P^{n}$, deformations of flat conformal structures do not arise from these choices. In this case, it seems that the conformal symmetries of $\mathbf{R} P^{n}$ absorb variations of these choices, just as an essential conformal transformation on $S^{n}$ absorbs a deformation arising from changing the length of a cylinder as we have seen in Remark 2 following Theorem 3.1.

\section{REFERENCES}

[1] B. N. Apanasov, Discrete Groups in Space and Uniformization Probrems, Math. and its Appl., Vol 40, Kluwer Academic Publ. Group, Dordrecht, Holland, 1991. MR 93h:57026

[2] R. Benedetti and C. Petronio, Lectures on Hyperbolic Geometry, Springer-Verlag, Berlin Heidelberg, 1992. MR 94e:57015 
[3] J. Cerf, Sur les difféomorphismes de la sphère de dimension trois $\left(\Gamma_{4}=0\right)$, Lecture Notes in Math. 53, Springer-Ferlag, Berlin Heidelberg, 1968. MR 37:4824

[4] W. M. Goldman, Conformally flat manifolds with nilpotent holonomy and the uniformization problem for 3-manifolds, Trans. Amer. Math. Soc. 278 (1983), 573-583. MR 84j:53043

[5] - Projective structures with Fuchsian holonomy, J. Diff. Geom. 25 (1987), 297-326. MR 88i:57006

[6] W. M. Goldman and Y. Kamishima, Conformal automorphisms and conformally flat manifolds, Trans. Amer. Math. Soc. 323 (1991), 797-810. MR 91e:53034

[7] J. Hempel, 3-manifolds, Annals of Math. Studies 86, Princeton Univ. Press, Princeton, New Jersey, 1976. MR 54:3702

[8] H. Izeki, On the decomposition of conformally flat manifolds, J. Math. Soc. Japan 45 (1993), 105-119. MR 93h:53036

[9] D. Johnson and J. J. Millson, Deformation spaces associated to compact hyperbolic manifolds, in Discrete Groups in Geometry and Analysis, Papers in Honor of G. D. Mostow on His Sixtieth Birthday, Progress in Math. 67 (R. Howe, eds.), Birkhäuser, Boston, Basel, 1987, pp. 48-106. MR 88j:22010

[10] N. H. Kuiper, On conformally flat spaces in the large, Ann. of Math. 50 (1949), 916-924. MR 11:133b

[11] N. H. Kuiper, On compact conformally Euclidean spaces of dimension > 2, Ann. of Math. 52 (1950), 478-490. MR 12:283c

[12] R. S. Kulkarni, On the principle of uniformization, J. Diff. Geom. 13 (1978), 109-138. MR 81k:53009

[13] R. S. Kulkarni and U. Pinkall, Uniformization of geometric structures with applications to conformal geometry, in Lecture Notes in Math. 1209 (1986), Springer-Verlag, Berlin Heidelberg, 190-209. MR 88b:53036

[14] A. G. Kurosh, The Theory of Groups, Vol. 2, Chelsea, New York, 1956. MR 18:188f

[15] J. Lelong-Ferrand, Transformations conformes et quasi-conformes des variété riemanniennes compactes (démonstration de la conjecture de A. Lichenerowicz), Acad. Roy. Beg. Sci. Mem. Coll. in $8^{\circ}$ (2) 39 (1971), no. 5. MR 48:1100

[16] J. Lelong-Ferrand, Geometrical interpretation of scalar curvature and regularity of conformal homeomorphism, in Differential Geometry and Relativity (1976), Reidel Publ. Co., Dordrecht, Holland, 91-105. MR 56:1222

[17] J. Milnor, Lectures on the h-Cobordism Theorem, Princeton Univ. Press, Princeton, New Jersey, 1965. MR 33:8352

[18] M. Obata, The conjectures on conformal transformations of Riemannian manifolds, J. Diff. Geom. 6 (1971), 247-258. MR 46:2601

[19] I. M. Singer, Infinitesimally homogeneous spaces, Comm. Pure Appl. Math. 13 (1960), 685-697. MR 24:A1100

[20] W. P. Thurston, The Geometry and Topology of Three-Manifolds, Princeton University Lecture Notes, 1979.

[21] M. Vuorinen, Conformal Geometry and Quasiregular Mappings, Lecture Notes in Math. 1319, Springer-Verlag, Berlin Heidelberg, 1988. MR 89k:30021

Graduate School of Polymathematics, Nagoya University, Chikusa-ku, Nagoya 46401, JAPAN

E-mail address: izeki@math.nagoya-u.ac.jp 